\title{
Continuous neural activations of embodied-linguistic representations in modality switching costs
}

\author{
Pei Liu ${ }^{1}$, Louise Connell ${ }^{1}$ and Dermot Lynott ${ }^{1,2}$ \\ ${ }^{1}$ Department of Psychology, Lancaster University \\ ${ }^{2}$ Department of Psychology, Maynooth University
}

\section{Author Note}

We have no conflicts of interest to declare. Correspondence concerning this article should be addressed to Louise Connell (1.connell@lancaster.ac.uk) or Dermot Lynott (dermot.lynott@mu.ie)

Word count: 873I 


\begin{abstract}
Modality switching costs (MSCs) are one of the classic effects that support the embodied views of conceptual representations. They refer to a delay in response time to verify a sensory property of a certain perceptual modality (e.g., visual: SUN - bright), when the previous sensory property has been of a different modality (e.g., auditory: BLENDER - loud) compared to a property of the same modality (e.g., visual: ROSE - red). Such costs indicate that conceptual representations require the recruitment of modality-specific resources. However, MSCs could also result from the distributional pattern of property words: the reason why loud $->$ bright takes longer than red -> bright could be because bright and loud do not co-occur in the same linguistic context as frequently as bright and red. In the present study, we examined how well MSCs were predicted by an embodied model (switch / no-switch between perceptual modalities) versus a linguistic model (switch / no-switch between linguistic distributional clusters), in behavioural (RT) and continuous event-related EEG potentials (ERP) paradigms. The behavioural data supported the linguistic model in explaining MSCs and found MSCs to be moderated by the target modality, the ERPs showed that linguistic distributional pattern played a crucial role in the neural activations of MSCs. What used to be found as a result of perceptual switching (e.g., "early N400" effect) could be better explained by the linguistic model. The embodied component was activated later than the linguistic component, accounting for activations associated with semantic representation (typically in N400 area). Later during processing, both components were active for decision making (often manifested as LPC).
\end{abstract}

Keywords: modality switching costs, embodied simulation, linguistic distributional pattern, EEG

Word count: 8731 words 
Continuous neural activations of embodied-linguistic representations in modality switching costs

Embodied cognition (also called grounded or situated cognition) in its most consensual form suggests that conceptual processing involves the simulation (i.e., partial reactivation) of sensory, motor, affective, introspective and other bodily experiences of concepts (Barsalou, 1999). When we process a sentence such as The sun is bright, we "relive" the bodily experiences with the sun and specifically the visual experience of its brightness. The modality switching costs (MSCs; Pecher, Zeelenberg, \& Barsalou, 2003) showed that people's response to a word pair such as $S U N$ - bright would be delayed if the previous word pair they verified had pertained to a different sensory modality (e.g., BLENDER - loud) compared to the same modality (e.g., ROSES - red). Such costs were interpreted as a result of engaging modality-specific resources. People were slower to respond to bright because their attention had been previously engaged in the auditory modality when processing loud. Further studies also found MSCs across linguistic and perceptual stimuli (Van Dantzig, Pecher, Zeelenberg, \& Barsalou, 2008) and during novel concept creation (Connell \& Lynott, 2011), again confirming that people "relive" the sensory experiences (e.g., "seeing" the sun's light, "hearing" a blender's noises) when they verify these concepts.

Meanwhile, the linguistic account of conceptual representation proposes that the meaning of a word is encoded (at least partially) in the distributional pattern of the linguistic symbols (Landauer \& Dumais, 1997). As Firth (1957) famously put it, "you shall know a word by the company it keeps" (p.11). Linguistic distributional patterns can encode relationship of objects, events and matters to a certain degree (Louwerse, 2011). The meaning of sun could be understood by its semantic neighbours such as sky, hot, bright, etc. This linguistic account provides another 
interpretation for MSCs. The reason why people were faster to respond to bright after red than after loud was that bright and red co-occurred in the same context more frequently than bright and loud. Indeed the co-occurrence frequencies could differentiate the sensory modality of perceptual words to some extent (Louwerse \& Connell, 2011). A corpus study illustrated that the co-occurrence frequencies of sensory property words (e.g., bright, loud, etc.) varied along three dimensions, which broadly corresponded to the perceptual property of vision-haptics, olfaction-gustation, and audition. Switch between the linguistic dimensions were capable of accounting for the faster responses of MSCs, while switch between the embodied dimensions were capable of accounting for the slower responses.

\section{Embodied-Linguistic Conceptual Representations}

Many researchers have explicitly stated that conceptual representations are both embodied and linguistic (Andrews, Vigliocco, \& Vinson, 2009; Barsalou, Santos, Simmons, \& Wilson, 2008; Connell \& Lynott, 2014; Louwerse \& Jeuniaux, 2008). Such a combinatorial account can bridge the gap between the two accounts, and thus help avoid the problems faced individually by either. At the linguistic end, the extreme form of this linguistic account needs to face the challenge of the grounding problem (Harnad, 1990), which questions how the linguistic symbols link to its real-world referents. If the meaning of a word is simply defined by other words, it is hard to say that any words have meanings at all. This problem is perfectly illustrated by Searle (1980)'s rendition Chinese room argument. Suppose a person who does not know Chinese sits in a closed room, and receives input in Chinese from a slot. He follows an instruction book that produces Chinese characters as response to the input. Suppose the instructions are sufficiently well written, and the responses so convincing that the person who gives the Chinese input thought the person in the room was really Chinese. However, the case remains that the person in the room 
does not know the meaning of any of the Chinese words he produces. In other words, simply knowing the distributional patterns between words is not the same as knowing a language. The combinatorial account allows words to be grounded in the brain systems of perception, action and emotion (Zwaan, 2014), though the degree to which each linguistic symbol is grounded is still a contended issue (Chemero, 2009; Harnad, 1990; Kintsch \& Dijk, 1978; Öttl, Dudschig, \& Kaup, 2017).

On the embodied side, the combinatorial account, among many of its benefits, affords a degree of flexibility to embodied simulation (Zwaan, 2014). As language comprehension does not only occur on the level of individual words and sentences, different representations are created based on the environment, context and goal of processing (Connell \& Lynott, 2014; Lebois, Wilson-Mendenhall, \& Barsalou, 2015; Louwerse \& Jeuniaux, 2010). One way that the linguistic component affords more flexible conceptual representation is by providing a shortcut for embodied simulation (Connell, 2018). Theories of embodied language processing, such as the Language and Situated Simulation (LASS; Barsalou et al., 2008) theory and the Symbol Interdependency theory (SIH; Louwerse, 2011), suggest that the linguistic component is more speedy and less costly, and thus peaks prior to the embodied component (although embodied simulation could be activated very quickly as shown by Hauk, Johnsrude, and Pulvermüller (2004) for example). Since the linguistic component can partially encode bodily experiences in the real world, it is useful as an indicator for whether future processing will be successful, in other words whether it is worth expending energy on the more costly embodied simulation (Connell \& Lynott, 2013).

Louwerse and Connell (2011) examined whether the MSCs could be accounted for by both simulation and linguistic information, and found that the linguistic component had indeed a temporal advantage. While a switch between the perceptual modalities (e.g., haptics $\rightarrow$ vision: warm $\rightarrow$ *bright) better accounted for 
the switching costs when people responded slowly, a switch between the linguistic dimensions (e.g., audition $\rightarrow$ haptic-vision: loud* $\rightarrow$ *bright*) better accounted for the costs when people responded quickly. In other words, people relied on the coarse-grained difference between the linguistic distributional patterns as a shortcut to enable more speedy response. A necessary condition for the linguistic component to be a shortcut is for the linguistic component to reach the peak of its activation before the embodied component. Support for this condition requires the examination of the continuous activations of both components during online processing, which is the aim of the present study.

\section{Present Study}

The present study sets out to test whether embodied and linguistic components both contribute to MSCs, and explore their activations during online processing using event-related EEG potentials (ERP). ERP is a segment of EEG waveforms time-locked to an event, which in the case of MSCs is the presentation of the property word (e.g., bright; Otten \& Rugg, 2005). ERP is often characterised as discrete components, which is a segment of waveform that is functionally significant, circumscribed to a certain time window. Previous studies have found many ERP components associated with MSCs, most prominent of which were N400 and P600 or late positivity complex (LPC; Bernabeu, Willems, \& Louwerse, 2017; Collins, Pecher, Zeelenberg, \& Coulson, 2011; Hald, Hocking, Vernon, Marshall, \& Garnham, 2013; Hald, Marshall, Janssen, \& Garnham, 2011). A perceptual modality switch often elicited a greater negativity around $400 \mathrm{~ms}$ from the property onset and greater positivity after $600 \mathrm{~ms}$ from the property onset, which was interpreted as a difference in semantic representation and decision making respectively.

However, the findings of these components have been highly inconsistent. The N400 effect alone was found in the posterior region in some cases (Bernabeu et al., 
2017; Hald et al., 2013), while in anterior region in others (Collins et al., 2011, Hald et al. (2011)). In some cases, it was found in the typical window around 400ms (Collins et al., 2011), while in others an earlier window from $270 \mathrm{~ms}$ to $370 \mathrm{~ms}$ (Bernabeu et al., 2017; Hald et al., 2011). Furthermore, different ERP components were also found to be associated with different perceptual modalities. According to Collins et al. (2011), the N400 effect was manifested only for visual properties, but in other studies it was found for properties of all sensory modalities. These inconsistencies are most likely due to the complex nature of semantic processing, which involves not only conceptual representation but from sublexical processing to integration of semantic representations (Hauk, 2016). These previous studies did not only examine the effect of modality switching but also other linguistic factors such as negated sentences, which could easily distort observed waveforms (Luck, 2005). Furthermore, these ERP studies so far have not considered the linguistic distributional patterns as a cause for MSCs. Therefore, the present study will focus on the switching costs alone, accounting for it by either a switch between perceptual modalities or between linguistic dimension.

To achieve a better control and maximise the effect, we conducted a behavioural study first with only visual, auditory and haptic words (Experiment 1). According to Louwerse and Connell (2011), the linguistic dimensions are coarser-grained than the perceptual modalities, that is while a linguistic switch is certainly a perceptual switch, a perceptual switch may not be a perceptual switch. For example, a switch between haptic and visual modalities (warm and bright) is a perceptual switch but a linguistic non-switch because haptic words and visual words belong to the same linguistic dimension. Thus, we are able to differentiate the effect of a perceptual switch from a linguistic switch. We hypothesised that both a perceptual switch and a linguistic switch could account for the switching costs in this experiment. From Experiment 1, we selected the stimuli that reliably produced 
either perceptual or linguistic MSCs (or both) to be used in Experiment 2, the ERP study. Our study will be largely exploratory because of the inconsistency in previous findings, and the inability to pinpoint the time windows and the scalp regions of the linguistic dimension switch. Thus, only tentative hypotheses could be proposed, which are 1 . the linguistic switch should affect the waveform prior to the embodied switch; 2. the embodied switch should mainly affect the waveform around 400ms.

\section{Experiment 1: Behavioural Modality Switching Costs}

By studying the modality switching costs, it is possible to identify the separate contributions of embodied and linguistic components to conceptual representation. Therefore, in this experiment, our aim is to examine the costs of perceptual modality switch and the costs of linguistic dimension switch separately. Participants will read pairs of concepts (nouns) and properties (adjectives), such as $S U N$ - bright, and decide whether the property is usually true for the concept. The properties shown will be of three perceptual modalities, vision, audition and haptics, but only two linguistic dimensions based on how the properties group together according to their distributional patterns. Therefore, we expect there to be a cost (i.e., delay in reaction time) when there is a switch between perceptual modalities, as well as between linguistic dimensions. In this section, we report how we determined our sample size, all data exclusions, all manipulations, and all measures in the study.

\section{Method}

Participants. Based on the effect size from Louwerse and Connell (2011) and requiring statistical power of .95 with an alpha level of .05, we estimated the required sample size to be 45 participants using $\mathrm{G}^{*}$ Power (Faul, Erdfelder, Buchner, \& Lang, 2009; Faul, Erdfelder, Lang, \& Buchner, 2007). Forty-eight native speakers 
of English were thus recruited for a payment of $£ 3.50$ or course credit (age: 19.82 (1.51); female: 30; right-handed: 39).

Materials. One hundred and fifty concept-property items, such as $S U N$ bright, were used in the present study. The property words were selected from the modality exclusivity norms of Lynott and Connell (2009) and Dantzig, Cowell, Zeelenberg, and Pecher (2011), in which the property words were rated according to how strongly they could be experienced by the five major sensory modalities, which are vision, audition, gustation, olfaction and haptics. The property words in the present study were all uni-modal, such that the difference between the ratings of the dominant modality (i.e., the modality with the highest rating) and the modality with the second highest rating was greater than 0.75 . These words pertained to three dominant modalities, which are vision $(N=60)$, audition $(N=60)$ and haptics $(N$ $=30$ ). They were paired with concept words, for which the properties were not only true but also salient (e.g., CARNATION - red instead of CARNATION - black, the latter of which could be true but was not salient), so that the concepts could activate the properties automatically. The co-occurrence frequencies between concepts and properties were below 100 million (Netspeak.com) to avoid high word association, and the words were known by more than $75 \%$ of the population.

The perceptual modality of the property words was simply the dominant modality. Furthermore, we figured out the linguistic dimension of the property words using a principle components analysis (PCA) of the co-occurrence frequencies. Initially, we collated a set of 244 property words (94 did not appear in the final material set because they did not satisfy the requirements in the previous paragraph), whose bi- to 5-gram frequencies were extracted from the Google Web-1T corpus (Brants \& Franz, 2006). The bi-gram frequency refers to the co-occurrence frequency between two words (e.g., mumbling and bright) with zero intervening words (i.e., mumbling bright or bright mumbling); the tri-gram frequency refers to 
that with one intervening word (i.e., mumbling $x$ bright or bright $x$ mumbling); and so forth for 4- and 5- gram. These frequencies of each pair of properties were summed and transformed logarithmically, resulting in a 244 x 244 matrix which was submitted to a PCA. After Varimax rotation with Kaissar normalisation, two principle components were extracted that could explain $61.25 \%$ of the total variance. PC1 accounted for $50.76 \%$ of the total variance; PC2 accounted for $10.49 \%$ extra of the total variance. The property words were thus allocated to either PC1 or PC2 based on which of the factor loadings was higher. We found that PC1 had a significant, positive correlation with the visual strengths of the properties (Table 1), and PC2 had a significant, positive correlation with the auditory strengths; while both components had a weak positive correlation with the haptic strengths.

Each target properties were paired with three different prime properties, so that every property appeared in all three switching conditions, which are a switch between both perceptual modalities and linguistic dimensions, a switch between perceptual modalities only but no switch between linguistic dimensions, or no switch between either perceptual modalities or linguistic dimensions. For instance, for the same target property bright, mumbling $->$ bright caused a switch between both perceptual modalities, that is from audition to vision, and linguistic dimensions, that is from $\mathrm{PC} 2$ to $\mathrm{PC} 1$; rough $->$ bright caused a switch only in perceptual modalities, that is from haptics to vision, but not between linguistic dimensions, both of which were of PC1; and colourful -> bright represented neither switch. A further list of 270 fillers was added, among which 45 were true and 225 were false. The true fillers were concept-property items that did not pertain to the three sensory modalities of interest; whereas false fillers were concept-property pairs in which the properties were not usually true for the concept (e.g., WHISKY - fluffy). Some of the false fillers were highly associated words (e.g., TEMPLE - praying), so that people could not decide the truthfulness of the concept-property items from the linguistic 
Table 1

Correlation between the PCs and modality strengths

\begin{tabular}{llllll}
\hline & PC1 & PC2 & Visual & Haptic & Auditory \\
\hline PC1 & 1.00 & .00 & $.64^{* *}$ & .12 & $-.61^{* *}$ \\
PC2 & - & 1.00 & $-.21^{* *}$ & .12 & $.31^{* *}$ \\
Visual & - & - & 1.00 & $.18^{* *}$ & $-.72^{* *}$ \\
Haptic & - & - & - & 1.00 & $-.42^{* *}$ \\
Auditory & - & - & - & - & 1.00 \\
\hline
\end{tabular}

Note. ${ }^{* *}$ means $\mathrm{p}<.01$

association of the words alone (Solomon \& Barsalou, 2004). The participants also saw 25 practice trials, using true items not featured in the main experiment.

In the experiment, each participant saw the items and fillers in a random order. The visual and auditory items were presented only once either as prime or as target, and the haptic items were presented twice as both prime and target, resulting in 90 prime $->$ target pairs for each participant. Across the experiment, all items were used as both prime and target, leading to 6 lists of stimuli in total (prime/target (2) x switching conditions (3)) to which participants were randomly assigned.

Procedure. The experiment was reviewed by Lancaster University's Department of Psychology Ethics Committee and approved by Lancaster University's Research Ethics Committee. After brief information about the study, the participants were sat normally from a PC screen. Participants received both verbal and written instructions (in the exact wording as reported) that they would see concept-property pairs on the screen, and the task was to decide whether the properties were usually true for the concepts. For example, if they saw CARNATION - red, the answer would be "yes"; whereas if they saw CARNATION 
- black, even though it was possible, it was not usually true, so the answer would be "no". All concepts were in the uppercase ("CARNATION") and properties in the lowercase ("red"). The concepts and the properties appeared one after another in black letters on a grey background (Figure 1).

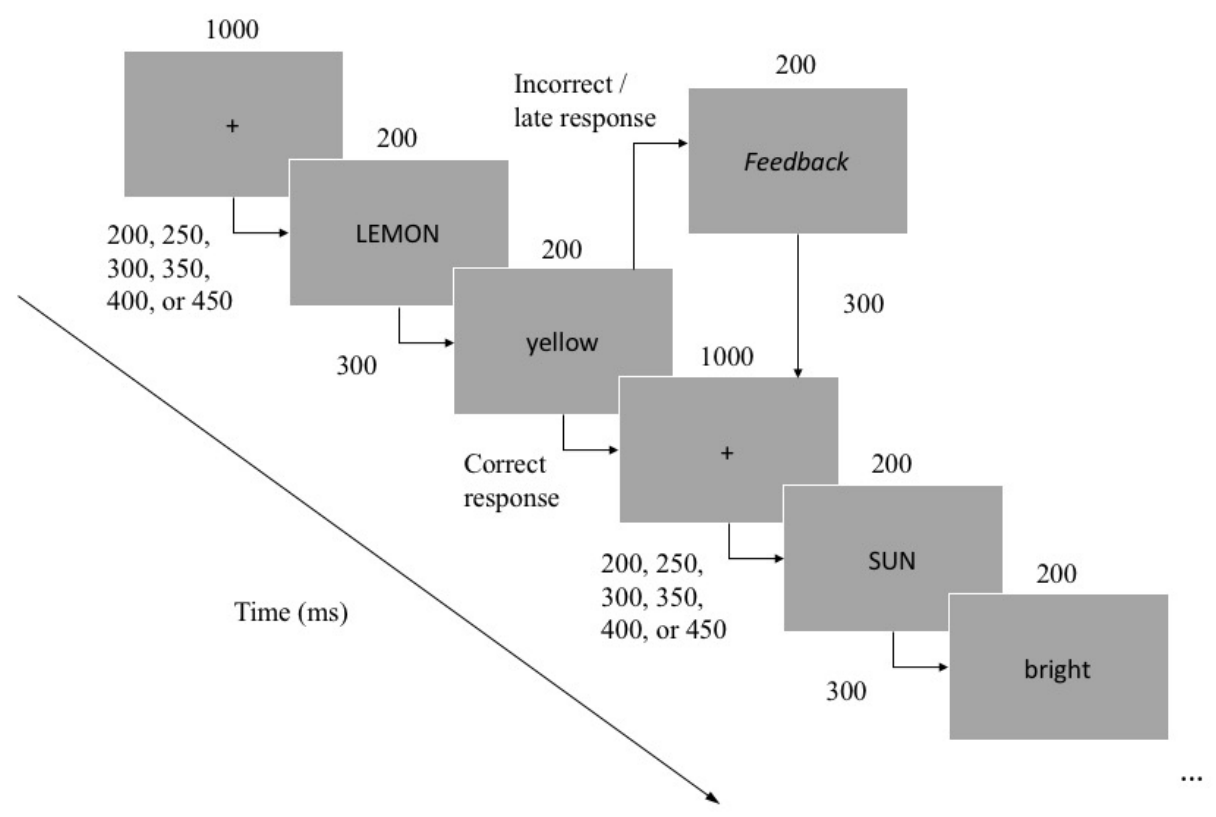

Figure 1. Sequence of presenting the CONCEPT-property pairs. Response timed out after $3000 \mathrm{~ms}$ after the property onset.

Figure 1 shows the structure of a concept-property item. The trial started with a fixation cross for $1000 \mathrm{~ms}$ followed by a blank screen of variable length $(200 \mathrm{~ms}$ to $450 \mathrm{~ms})$. Then the concept and the property appeared consecutively each for $200 \mathrm{~ms}$ with a blank of $300 \mathrm{~ms}$ in between. Once the property word appeared, participants could start to respond by pressing the comma key (",") for "yes" or the full stop key (".") for "no". If the response were incorrect, the participants would receive feedback ("Error"). If they failed to respond within a 3-second interval after the onset of the property words, they would receive a feedback (“***TOO $\left.\mathrm{SLOW}^{* * *} "\right)$. The accuracy and reaction time were recorded. Afterwards, the next trial automatically started. 
Analysis and design. Data were analysed and reported using $\mathrm{R}^{1}$. Two linear mixed-effect (LME) models were separately built for each type of switch, one for the perceptual modality switch and one for the linguistic dimension switch. The dependent variable was the reaction time (RT) to the target concept-property items from the target property onset. Participants and items were crossed random variables to predict random intercepts, ${ }^{2}$ and the target modality (visual, auditory

${ }^{1}$ We used R (Version 3.5.0; R Core Team, 2017) and the R-packages abind (Version 1.4.5; Plate \& Heiberger, 2016), arm (Version 1.10.1; Gelman \& Su, 2018), BayesFactor (Version 0.9.12.4.2; Morey \& Rouder, 2015), bookdown (Version 0.7; Xie, 2016), broom (Version 0.4.4; Robinson, 2017), coda (Version 0.19.1; Plummer, Best, Cowles, \& Vines, 2006), contrast (Version 0.21; Kuhn, Steve Weston, Wing, Forester, \& Thaler, 2016), data.table (Version 1.11.4; Dowle \& Srinivasan, 2017), doBy (Version 4.6.1; Højsgaard \& Halekoh, 2016), dplyr (Version 0.7.5; Wickham, Francois, Henry, \& Müller, 2017), Formula (Version 1.2.3; Zeileis \& Croissant, 2010), ggplot2 (Version 2.2.1; Wickham, 2009), gridExtra (Version 2.3; Auguie, 2017), Hmisc (Version 4.1.1; Harrell Jr, Charles Dupont, \& others., 2018), interplot (Version 0.1.5; Solt \& Hu, 2015), knitr (Version 1.20; Xie, 2015), lattice (Version 0.20.35; Sarkar, 2008), lme4 (Version 1.1.17; Bates, Mächler, Bolker, \& Walker, 2015), lmerTest (Version 3.0.1; Kuznetsova, Brockhoff, \& Christensen, 2017), magrittr (Version 1.5; Bache \& Wickham, 2014), MASS (Version 7.3.50; Venables \& Ripley, 2002), Matrix (Version 1.2.14; Bates \& Maechler, 2017), mgcv (S. N. Wood, 2003, 2004, Version 1.8.23; 2011; S. Wood, N., Pya, \& S"afken, 2016), multcomp (Version 1.4.8; Hothorn, Bretz, \& Westfall, 2008), mvtnorm (Version 1.0.8; Genz \& Bretz, 2009), nlme (Version 3.1.137; Pinheiro, Bates, DebRoy, Sarkar, \& R Core Team, 2017), papaja (Version 0.1.0.9735; Aust \& Barth, 2017), plyr (Wickham, 2011; Version 1.8.4; Wickham et al., 2017), psych (Version 1.8.4; Revelle, 2018), purrr (Version 0.2.5; Henry \& Wickham, 2018), rcartocolor (Version 0.0.22; Nowosad, 2017), rms (Version 5.1.2; Harrell Jr, 2018), SparseM (Version 1.77; Koenker \& Ng, 2017), survival (Version 2.42.3; Terry M. Therneau \& Patricia M. Grambsch, 2000), TH.data (Version 1.0.8; Hothorn, 2017), and tidyr (Version 0.8.1; Wickham \& Henry, 2017) for all our analyses and the writing up of this manuscript. 2 Although model including participants as the only random intercept explained the data worse than the empty model by 843.08 times $\left(B F_{10}=0.001\right)$, model with participants and item as crossed random intercepts better explained the data than the model with only participants as random intercept 35.47 times $\left(B F_{10}=35.47\right)$. 
and haptic, with auditory target as the reference level) and the switching condition (switch coded as 1, non-switch coded as 0 ), plus their interactions were the fixed predictors. Planned comparisons for each target modality were also run, that is perceptual/linguistic switch versus non-switch for the three target perceptual modalities separately. $p$-values for the planned comparisons were corrected using Tukey's HSD (reported in Supplementary Materials).

To examine the effect of switching costs and its interaction with target modality, we conducted model comparisons in a hierarchical fashion. For perceptual modality switch:

Step 1: Models with random predictors and target modality only;

Step 2: Models with random predictors and target modality + perceptual modality switch;

Step 3; Models with random predictors and target modality + perceptual modality switch and their interaction.

For linguistic dimension switch, the same three steps were carried out with linguistic dimension switch instead of perceptual modality switch.

In our study, all model comparisons were conducted using Bayes factors (BF), which denotes the ratio of likelihood of the data under both null and alternative hypothesis (Jarosz \& Wiley, 2014). If the $\mathrm{BF}$ of $\mathrm{H}_{1}$ against $\mathrm{H}_{0}$ (i.e., $B F_{10}$ ) is 5 , it means that the data is 5 times more likely to occur under $\mathrm{H}_{1}$ than $\mathrm{H}_{0}$. BF is superior to the $p$-value in two ways. First, BF could inform us how confident we can be with the hypothesis. We can be more confident with the alternative hypothesis if $B F_{10}=100$ than if $B F_{10}=10$. In contrast, $p$-values do not bear any significance to the power of the study, the size of the effect or the quality of the hypothesis (see Wagenmakers, 2007 for detailed discussion concerning $p$-values). Second, we do not need to conduct post hoc corrections of multiple comparisons with BF. In the 
Table 2

Interpretation of BF as evidence

for $H 1$

\begin{tabular}{ccc}
\hline$B F_{01}$ & $B F_{10}$ & Support for $H_{1}$ \\
\hline $1-.33$ & $1-3$ & Anecdotal \\
$.33-.10$ & $3-10$ & Substantial \\
$.10-.03$ & $10-30$ & Strong \\
$.03-.01$ & $30-100$ & Very strong \\
$<.01$ & $>100$ & Decisive \\
\hline
\end{tabular}

present paper (especially in Experiment 2), a large number of comparisons need to be conducted for the same set of data, which causes the inflation of Type I error. BF eliminates this concern by presenting the relative likelihood of null and alternative hypothesis in each test. As a result, we will focus on BFs in this paper, only reporting the regression coefficients and BFs in the manuscript, while the $p$-values and all other results from null-hypothesis significance testing will be included in the Supplementary Materials.

The BF of LME can be easily obtained in R by extracting the Bayesian information criteria $(\mathrm{BIC}) . B F_{01}$ (comparing $\mathrm{H}_{0}$ against $\mathrm{H}_{1}$ ) is calculated as:

$$
B F_{01}=e^{\frac{\left(B I C\left(H_{1}\right)-B I C\left(H_{0}\right)\right)}{2}}
$$

$B F_{10}$, which denotes the likelihood of $\mathrm{H}_{1}$ against $\mathrm{H}_{0}$ is simply the inverse of $B F_{01}$. While the continuous scale of $\mathrm{BF}$ is useful enough, we could conveniently describe the results, using Jeffreys (1998)'s guideline to characterise BF as Table 2. 


\section{Results and Discussion}

Seven participants' data were excluded from analysis because their accuracy to the fillers was too low $(<65 \%)$. No further participants were excluded because no one's RT was exceptionally long (i.e., $>2.5 \mathrm{SD}$ from the group mean). 849 prime-target pairs $(23.01 \%)$ were excluded because the response to either or both items were incorrect (519 targets (14.07\%) and 412 (11.17\%) primes). Finally, we removed individual target items whose $\mathrm{RT}$ were more than 2.5SD from the condition means, which led to further exclusion of 70 trials $(2.46 \%)$. Thus, 2771 trials were left and entered into the analysis.

The grand mean of RT was $805 \mathrm{~ms}(S D=255 \mathrm{~ms})$ across all three target modalities. Condition means of each modality were $805 \mathrm{~ms}$ (256ms) for auditory targets, $781 \mathrm{~ms}(247 \mathrm{~ms})$ for haptic targets, and $820 \mathrm{~ms}(259 \mathrm{~ms})$ for visual targets.

Perceptual switching costs. As seen in Figure 2, people reacted slower to the perceptual switch condition than the perceptual non-switch condition. However, the mixed-linear model did not support the modality switching costs. The regression coefficients of models at each step is shown in Table 3. A calculation of BFs showed that the data was no better explained by the Step 2 model (with target modality + perceptual switch) than by the Step 1 model (with target modality only; $B F_{21}=$ 1.35), nor was it better explained by the Step 3 model (with interaction) than the Step 2 model $\left(B F_{32}=2.20\right)$. In all, having the perceptual switch with its interaction with target modality explained the data 2.96 times better than the model with only random variables, providing only anecdotal evidence for the perceptual switching costs. Planned comparisons per target modality did not show a significant effect of perceptual switch in any perceptual modalities (Auditory $b=26.86$, Haptic $b=$ 3.70, Visual $b=15.00)$. 
Table 3

Regression coefficients of perceptual switching models in hierarchical model comparison

b $B F_{10}$

Step 1

Target modality

Haptic - auditory

Visual - auditory

27.56

Step 2

Target modality

Haptic - auditory

Visual - auditory 27.57

Perceptual switch

Step 3

Target modality

$$
\begin{array}{ll}
\text { Haptic - auditory } & -7.76 \\
\text { Visual - auditory } & 35.37
\end{array}
$$

$\begin{array}{ll}\text { Perceptual switch } & 26.84\end{array}$

Target modality : perceptual switch

Haptic-auditory : perceptual switch $\quad-23.14$

Visual - auditory : perceptual switch $\quad-11.84$ 


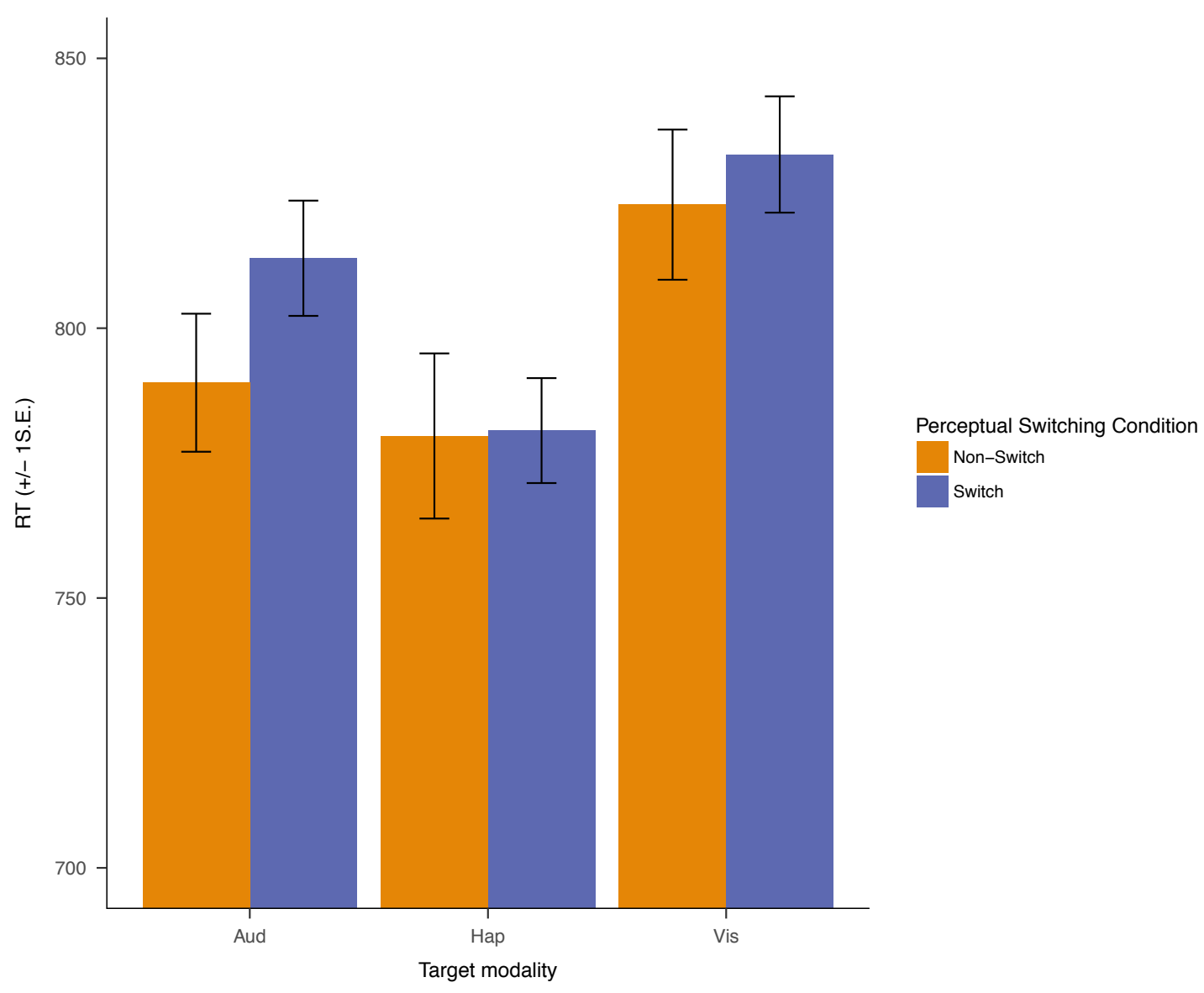

Figure 2. Mean RT of target trials per perceptual switching condition. Error bars denote $+/-1$ standard error.

Linguistic switching costs. Similar to the perceptual switching condition, the linguistic switching condition also produced a general trend as predicted, apart from visual items which was faster for a linguistic switch $(825 \mathrm{~ms}, \mathrm{SD}=264 \mathrm{~ms})$ than a non-switch $(831 \mathrm{~ms}, \mathrm{SD}=257 \mathrm{~ms}$; Figure 3$)$. The regression coefficients are shown in Table 4. Nevertheless, the BF comparing showed that although the data was no more likely under the Step 2 model than Step 1 model $\left(B F_{21}=0.90\right)$, it was 5.88 times more likely under the Step 3 model than Step 2 model, constituting substantial evidence for the interaction between linguistic dimension switch and target modality. Planned comparisons did not show a significant linguistic switching costs in any 


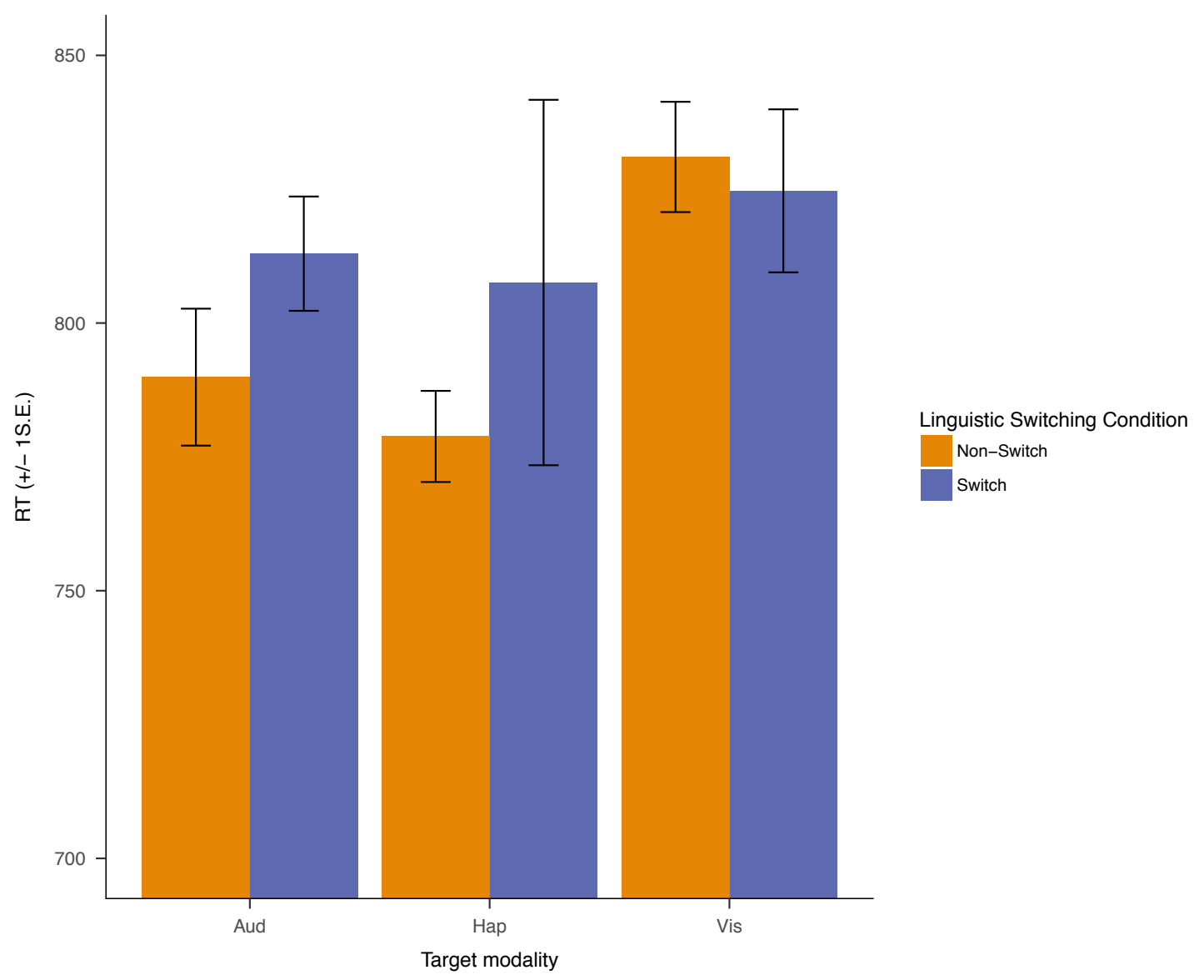

Figure 3. Mean RT of target trials per linguistic switching condition. Error bars denote $+/-1$ standard error.

perceptual modality (Auditory $b=23.06$, Haptic $b=28.75$, Visual $b=-6.33$ ).

Further comparisons between the perceptual switching models and linguistic switching models showed that these models were on the par in explaining the modality switching costs. The Step 2 model of perceptual switch (without the interaction term) was equal to that of linguistic switch $\left(B F_{P L 2}=1.50\right)$, while the Step 3 model of perceptual switch (with the interaction term) was not worse than that of the linguistic switch either $\left(B F_{P L 3}=0.56\right)$.

Summary. The BFs showed some anecdotal evidence in favour of perceptual modality switching costs which interacted with the target modality, as well as 
Table 4

Regression coefficients of linguistic switching models in hierarchical model comparison

\begin{tabular}{|c|c|c|}
\hline & $b$ & $B F_{10}$ \\
\hline \multicolumn{3}{|l|}{ Step 1} \\
\hline \multicolumn{3}{|l|}{ Target modality } \\
\hline Haptic - auditory & -22.99 & \\
\hline Visual - auditory & 27.56 & \\
\hline Step 2 & & 0.90 \\
\hline \multicolumn{3}{|l|}{ Target modality } \\
\hline Haptic - auditory & -16.37 & \\
\hline Visual - auditory & 31.23 & \\
\hline Linguistic switch & 11.17 & \\
\hline Step 3 & & 5.88 \\
\hline \multicolumn{3}{|l|}{ Target modality } \\
\hline Haptic - auditory & -6.65 & \\
\hline Visual - auditory & 47.87 & \\
\hline Linguistic switch & 26.87 & \\
\hline \multicolumn{3}{|l|}{ Target modality : linguistic switch } \\
\hline Haptic-auditory : linguistic switch & -5.58 & \\
\hline Visual - auditory : linguistic switch & -34.81 & \\
\hline
\end{tabular}


substantial evidence in favour of the interaction between linguistic dimension switching costs and the target modality. Specifically, target modality moderated the effect of the modality switching costs for both types of switch, such that the perceptual switching costs diminished for haptic items, whereas the linguistic switching costs were reversed for visual items.

\section{Experiment 2: ERP Effects of Modality Switching Costs}

In Experiment 1, we found some evidence for both perceptual and linguistic switching costs, though neither were very strong. What was of more importance was that the two types of switch led to switching costs in different ways (i.e., in different modalities). Therefore, it was ever more crucial to study how the switch would affect the neural activities during online processing. In this experiment, we studied the ERP of modality switching costs in a 1000ms epoch across the whole scalp. We proposed some tentative hypotheses which suggested that the perceptual modality switch should affect the ERP around 400ms after the property onset, and the linguistic dimension switch should affect the ERP prior to that time window. Here we report how we determined our sample size, all data exclusions, all manipulations, and all measures in the study.

\section{Method}

Participants. Twenty-five native speakers of English were recruited from Lancaster University for the payment of $£ 7 /$ hour (or the equivalent of credits). The sample size was determined based on Hald et al. (2011)'s study. Two participants were later excluded because of their high error rate to the filler trials (accuracy $<$ $65 \%)$. Among the rest of the participants, mean age was $21.00(S D=1.38)$, three were male and two were left handed. 
Materials. The prime $\rightarrow$ target pairs from Experiment 1 remained for this study if they resulted in a modality switching costs (i.e., the RT to a target property in a switch trial was longer than its RT in a non-switch trial), which were 114 different prime $\rightarrow$ target pairs. Twenty-two distinct auditory words and 21 visual words were chosen as targets, while the haptic targets were all removed because the remaining sample size was too small. Nineteen auditory properties, fourteen haptic properties and nineteen visual properties were chosen as prime items. All auditory property words (both primes and targets) were of PC1; 15 visual words were of PC1 and 25 PC2; as for haptic words (only primes) 5 were of PC1 and 9 were of PC2. All auditory targets were either both linguistic and perceptual switch (different prime $\rightarrow$ target pairs $N=36)$, or both linguistic and perceptual non-switch $(N=22)$; whereas visual targets could represent three different conditions: both switch $(N=$ 17), both non-switch $(N=19)$, and perceptual switch but linguistic non-switch $(N$ $=20)$.

All prime-target pairs were presented in a random order to each participant, as well as fillers which appeared in Experiment 1. Both prime $->$ target pairs and fillers were presented either once or twice in the whole experiment to boost the number of trials in each condition, resulting in 137 prime-target pairs and 237 filler pairs for each participant. The experiment also included a practice session of 10 trials which did not feature in the test phase.

Procedure. Participants were tested individually in a quiet room. They were seated at a distance away from the computer screen where they could read the words on the screen and rest their both hands on the desk to press the buttons. The trial began with a fixation cross (1 second) followed by a variable blank screen between 400ms and 1600ms. Afterwards, participants saw the concept-property pairs on the screen consecutively (i.e., 200ms word followed by $300 \mathrm{~ms}$ blank). Unlike Experiment 1, after a property disappeared, participants were required to refrain 
from response for 1 second until a prompt ("?") appeared, to make sure that the ERP would not be contaminated by the response readiness potential (Dehaene et al., 1998). If they had responded before the prompt appeared, a warning would appear ("Too fast. Please wait for the prompt '?'."), and the trial was dismissed. Once the prompt appeared, participants again needed to judge whether the property was usually true for the concept by pressing the "n" key (masked by a red sticker) with their left hand for "yes", or the "m" key (masked by a white sticker) with their right hand for "no". The same feedback for slow (>3s) or incorrect response was given as in Experiment 1.

Participants were asked not to blink or move freely, but were given specific time for such muscle movements. Between trials, participants were free to blink for 1 second, and they were told not to blink within each trial when the words were showing. They were also given self-administered break nine times during the experiments after each five-minute block. Furthermore, each block began with 4 seconds of blank screen for the EEG recording to be recalibrate, during which participants were free to blink.

Apparatus. Words were presented on a 19-inch CRT monitor operating at $100 \mathrm{~Hz}$ refresh rate using EPrime 1.0, and behavioural responses were recorded using a QWERTY keyboard. High-density EEG was recorded continuously using Hydrocel Geodesic Sensor Nets (Electrical Geodesics Inc., Eugene, OR, USA) at 128 locations referenced to the vertex $(\mathrm{Cz})$ online. The ground electrode was at the rear of the head (between $\mathrm{Cz}$ and $\mathrm{Pz}$ ). Electrophysiological signals were acquired at the sampling rate of $1000 \mathrm{~Hz}$ by an Electrical Geodesics Inc. amplifier with a band-pass filter of $0.1-100 \mathrm{~Hz}$. The impedances were checked prior to the beginning of the recording and they were considered acceptable if lower than $50 \mathrm{k} \Omega$.

EEG analysis. The digitized EEG was further processed offline using NetStation v 4.5.4. It was band-pass filtered between $0.4-100 \mathrm{~Hz}$ and was segmented 
into epochs including $200 \mathrm{~ms}$ before adjective onset and $1000 \mathrm{~ms}$ after adjective onset for the target trials. EEG epochs were automatically rejected for body and eye movements, as well as signals exceeding $\pm 200 \mu \mathrm{V}$ at any electrode. Data were further checked through visual inspection for eye-movements, eye-blinks and other artifacts not detected by the automated algorithm. The artifact free segments were baseline-corrected with respect to the average amplitude in the $200 \mathrm{~ms}$ window preceding the stimulus onset, and were re-referenced to the average reference. Then, they were exported to comma-separated value (csv) files per participant per trial and analysed in R.

In order to explore the continuous activations of embodied and linguistic components, we analysed the EEG output throughout the whole 1-second epoch after the adjective onset, and across the whole scalp (Amsel, 2011; Hauk, Pulvermüller, Ford, Marslen-Wilson, \& Davis, 2009; Hendrix, Bolger, \& Baayen, 2017). To limit the researcher degrees of freedom in choosing the time window and scalp region (J. P. Simmons, Nelson, \& Simonsohn, 2011), the EEG output per participant per stimulus were sliced into 20 uniformed segments of 50ms; ninety electrodes covering the region of a 20-20 EEG net were analysed one by one (Appendix B). The resulting data were averaged over each segment per electrode per participant per stimulus and were analysed in linear mixed-effect models (LME), instead of the conventional test of ANOVA which commits the language-as-fixed-effect fallacy (Clark, 1973). The analyses were exceptionally computationally heavy and thus were carried out distantly using the High End Computing Cluster (HEC; http://www.lancaster.ac.uk/iss/services/hec/) of Lancaster University.

Separate LME models were built to study perceptual modality switch and linguistic dimension switch. The models were built in a hierarchical fashion as Experiment 1. 
1. To examine the effect of perceptual modality switch, models were built in the following steps:

Step $1_{\mathrm{P}}$ : Models with random predictors and target modality (auditory coded as the reference level);

Step $2_{\mathrm{P}}$ : Models with random predictors and target modality + perceptual switch (non-switch coded as 0 , switch coded as 1);

Step $3_{\mathrm{P}}$ : Models with random predictors and target modality + perceptual switch and their interaction.

2. To examine the effect of linguistic dimension switch, same three steps were carried out:

Step $1_{\mathrm{L}}$ : Models with random predictors and target modality (auditory coded as the reference level);

Step $2_{\mathrm{L}}$ : Models with random predictors and target modality + linguistic switch (non-switch coded as 0 , switch coded as 1 );

Step $3_{\mathrm{L}}$ : Models with random predictors and target modality + linguistic switch and their interaction.

The models in each step were compared with the model in previous step using BF. Thus, the comparison between, for example, Steps $2_{\mathrm{P}}$ with Steps $1_{\mathrm{P}}$ showed how much more likely the data would be when the perceptual switch was considered than when it was not considered. In addition, we examined whether the data was more likely under the linguistic switch or perceptual switch models by comparing the the two Step 2 models above, as well as the two Step 3 models above.

\section{Results}


Data exclusion. Two participants were deleted because their accuracy to filler trials were lower than $65 \%$. Individual trials were removed if the response to either the prime or the target or both was incorrect (42 trials, 1.52\%). This left us 2713 trials, among which 1248 were auditory targets and 1465 were visual targets. For all auditory targets, 725 were both perceptual and linguistic switch (58.09\%), and 523 were both perceptual and linguistic non-switch (41.91\%); and for visual targets, 499 were both perceptual and linguistic switch (34.06\%), 477 were perceptual switch but linguistic non-switch (32.56\%), and 489 were both non-switch $(33.38 \%)$.

Behavioural analysis. We analysed the RT (descriptive statistics in Table 5) in LME models using hierarchical model comparison in the same way as Experiment 1. We found that the data could be best explained by the model with target modality only. In terms of perceptual switch, the target-modality model was 1.43 times better than the model with target modality and perceptual switch without the interaction term $\left(B F_{21}=0.70\right)$ and 1.17 times better than the model with the interaction term $\left(B F_{31}=0.86\right)$. In terms of linguistic switch, the target-modality model was 2.17 times better than the model without the interaction term $\left(B F_{21}=0.46\right)$ and 1.16 times better than the model with the interaction term $\left(B F_{31}=0.86\right)$. According to the target-only model from Step 1, response to visual targets were faster than auditory targets $(b=-0.60)$

Table 5

Mean (SD) of RT per condition in Experiment 2

\begin{tabular}{lcccc}
\hline & \multicolumn{2}{c}{ Perceptual } & \multicolumn{2}{c}{ Linguistic } \\
\cline { 2 - 5 } & Switch & Non-switch & Switch & Non-switch \\
\hline Auditory & $347(195)$ & $348(204)$ & $347(195)$ & $348(204)$ \\
Visual & $354(216)$ & $334(198)$ & $355(220)$ & $343(206)$ \\
\hline
\end{tabular}




\section{ERP analysis.}

\section{Overview.}

The mean BF across the scalp for the model with linguistic switch in Step 2 (i.e., without interaction) was $0.004(S D=0.003)$, and the mean $\mathrm{BF}$ for the model with perceptual switch in Step 2 was $0.005(S D=0.004)$, both suggesting very strong evidence for the null hypothesis (i.e., model with target modality only). Figure 4 shows the mean $B F_{21}$ across the scalp over time. The BFs were less than 0.33 for the whole epoch, and below 0.1 from 100ms after the target adjective onset. By comparing between perceptual and linguistic switch, it seemed that the perceptual switch had an advantage over the linguistic switch at the first 50ms of the epoch and around 300ms, whereas the linguistic switch had an advantage around $650 \mathrm{~ms}$. The same pattern was found for the models of interaction (i.e., data strongly favoured models with only linguistic or perceptual switch (Step 2) against models with interaction terms (Step 3; see Supplementary Materials)). Viewing across the scalp, we found overall evidence against both perceptual and linguistic switch. However, it was still possible that these switches affected ERP in a localised manner, which will be examined next.

\section{Perceptual switch.}

Figure 5a showed the topoplot of the evidence in favour of (or against) models with target modality and perceptual switch $($ Step $2 \mathrm{P})$ compared with target-modality models $\left(\right.$ Step $\left.1_{\mathrm{P}}\right)$; Figure $5 \mathrm{~b}$ showed the comparison between models with the interaction term between target modality and perceptual switch (Step $3_{\mathrm{P}}$ ) and models without the interaction term $($ Step $2 \mathrm{P})$. There was no widespread effect of perceptual modality switching costs or its interaction with target modality, apart from the beginning of the epoch (i.e., $0 \mathrm{~ms}$ to $50 \mathrm{~ms}$ ) in the occipital electrodes (e.g., E70, $\left.B F_{21}=7.14\right)$, that is the data was 7.14 times more likely under the model with 


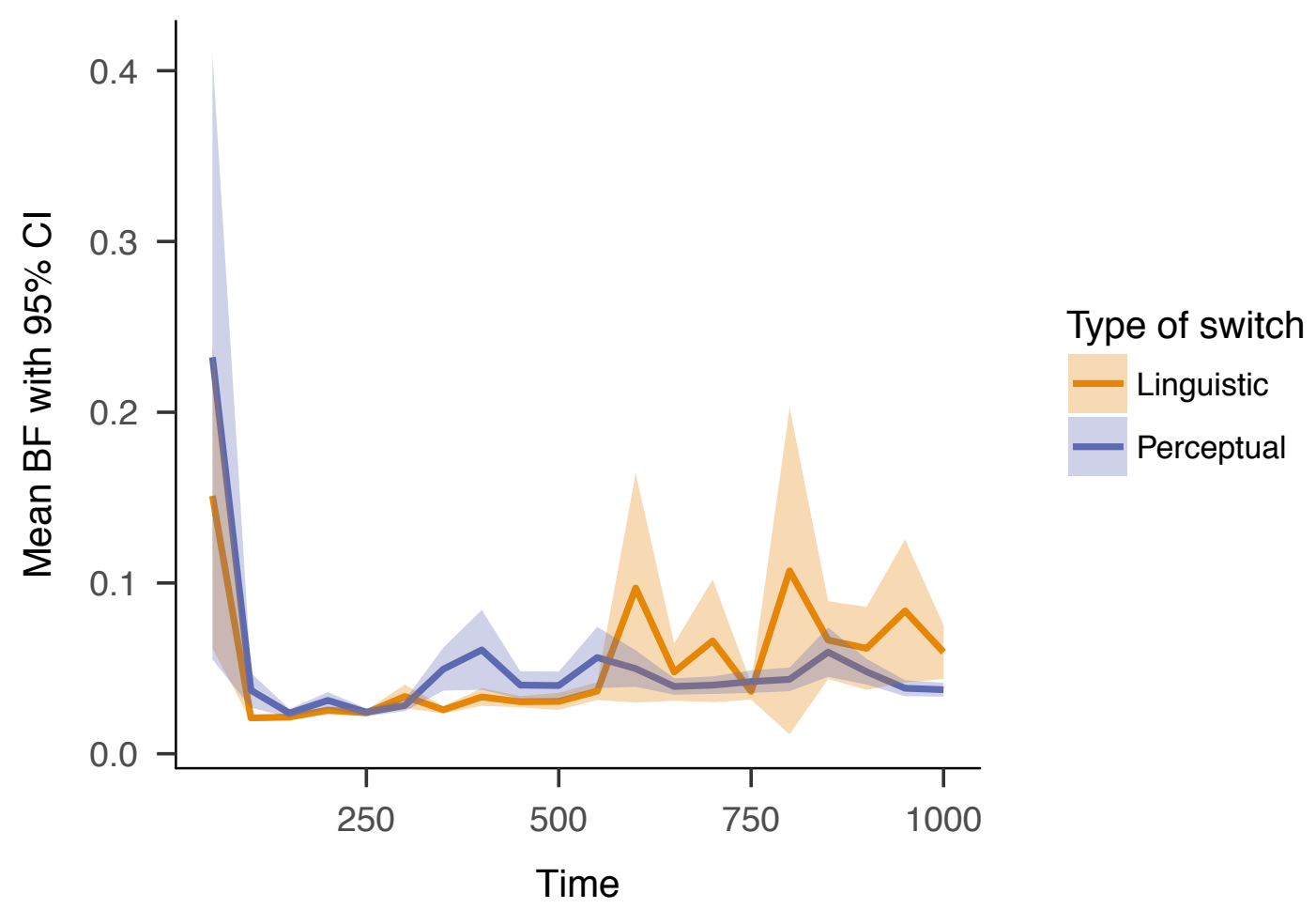

Figure 4. Line plot of mean $\mathrm{BF}_{21}$ with $95 \%$ credibility interval of perceptual and linguistic switch as a result of Step 2 across scalp at each 50ms slice.

perceptual modality switch than without. During this time, there was evidence that perceptual switching costs made the amplitude more negative $(b=-0.98)$. This effect was not moderated by the target modality, meaning that this early effect of perceptual switching costs had an effect on both visual and auditory targets equally.

Furthermore, the data suggested that in the following areas the interaction models were more likely than models excluding the interaction term (comparison between Step $3_{\mathrm{P}}$ and Step $2_{\mathrm{P}}$ models, not shown in topography). Perceptual switch interacted with the target modality between $300 \mathrm{~ms}$ and $350 \mathrm{~ms}$ in the frontal region (e.g., E19, $B F_{32}=19.70$, perceptual switch $b=1.81$, target modality $b=1.31$, interaction $b=-2.57)$. That is to say, in the frontal region, the perceptual switch was associated with more positivity than non-switch for auditory targets; but perceptual switch was associated with more negativity for visual targets. The 
opposite pattern was found in the right parietal region (e.g., E92, $B F_{32}=11.86$, perceptual switch $b=-1.26$, target modality $b=-1.13$, interaction $b=1.88$ ), which suggested that perceptual switch led to more negativity for auditory targets but more positivity for visual targets. Both of these effects coincided with previous findings of the early N400 effect (parietal effect with Bernabeu et al., 2017; frontal effect with Collins et al., 2011; Hald et al., 2011), which could be interpreted as a precursor of semantic processing.

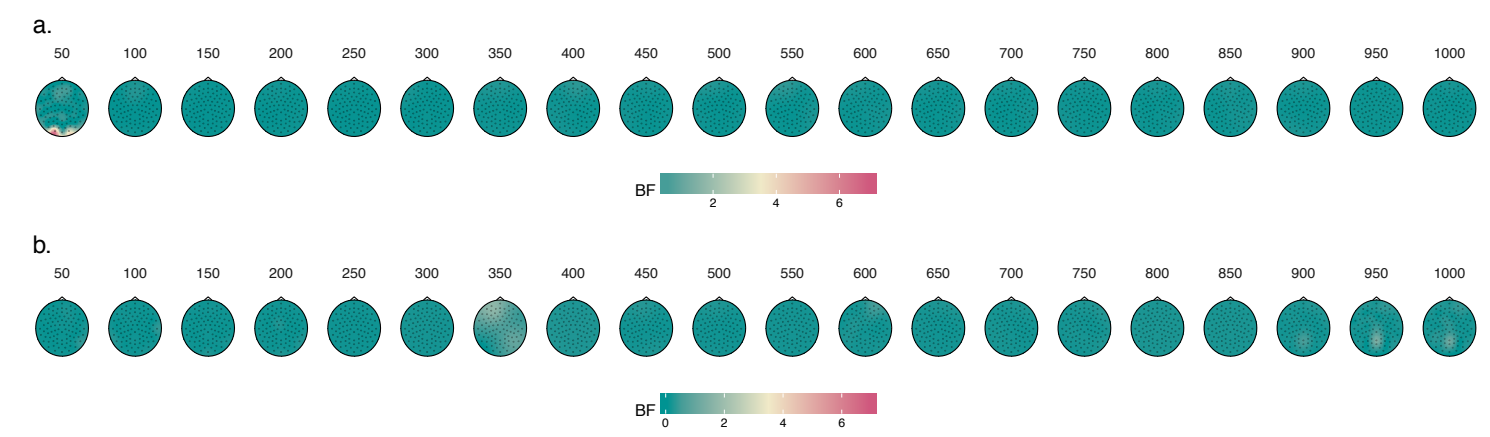

Figure 5. Effects of perceptual switch as a result of model comparisons between a. Step 2: models including perceptual switch vs Step 1: models excluding perceptual switch and b. Step 3: models including the interaction between perceptual switch and target modality vs Step 2: models excluding the interaction. $B F_{10}>3$ constitutes substantial evidence in favour of the perceptual switching costs.

\section{Linguistic switch.}

Evidence for linguistic dimension switching costs was found in similar regions as the perceptual modality switching costs (Figure 6). The data substantially favoured the linguistic modality switching costs (Step $2_{\mathrm{L}}$ compared with Step $1_{\mathrm{L}}$ ) at the onset of the epoch in the left occipital region as well (e.g., E70, BF21 = 3.17), such that the linguistic switch trial was associated with more negative amplitude $(b$ $=-0.92)$.

The data also strongly favoured the interaction between linguistic dimension 
switching costs and target modality between $300 \mathrm{~ms}$ and $350 \mathrm{~ms}$ at the left frontal (Step $3_{\mathrm{L}}$ compared with Step $2_{\mathrm{L}}$; e.g., E19, $B F_{32}=238.31$, linguistic switch $b=$ 1.81, target modality $b=1.23$, interaction $b=-3.07$ ) and right parietal regions (e.g., E92, $B F_{32}=167.36$, linguistic switch $b=-1.26$, target modality $b=-1.06$, interaction $b=2.27)$. In other words, similar to the perceptual switching costs, the linguistic switch was associated with more positivity for auditory targets but more negativity with visual targets in the frontal region; and the reversed pattern for the parietal region. For both regions, the data was more than 150 times more likely under models with the interaction than the models without interaction.

However, it is important to note that Step 3 models with the interaction term was not better than the Step 1 models which included only target modality. Take the linguistic models for example, when models in Step $2_{\mathrm{L}}$ was compared with Step $1_{\mathrm{L}}$, the data of E19 was 0.004 times more likely (i.e., 250 times less likely) under the Step $2_{\mathrm{L}}$ models which considered the linguistic switching costs alone without the interaction. In other words, the Step $3_{\mathrm{L}}$ model was 0.95 times more likely than the Step 1 model $\left(B F_{32} \times B F_{21}=238.31 \times 0.004=0.94\right)$. In the same way, the data of E92 was 0.002 times more likely (i.e., 500 times less likely) under the Step $2_{\mathrm{L}}$ model than the Step $1_{\mathrm{L}}$ model, so the Step $3_{\mathrm{L}}$ model was 3 times worse than the Step $1_{\mathrm{L}}$ model $\left(B F_{31}=0.33\right)$.

\section{Comparison between perceptual and linguistic switch.}

From the hierarchical model comparison, we discovered that perceptual switch and linguistic switch shared similar patterns of activities, supporting their effects at the occipital region at the onset of the epoch, and their interaction with target modality at both the left frontal region and the right parietal region between $300 \mathrm{~ms}$ and $350 \mathrm{~ms}$. Therefore, it is worthwhile to examine which type of switch was supported more strongly by the data. Figure 7 showed the evidence in favour of one type of switch against the other (i.e., comparison of Step 2 models and comparison 


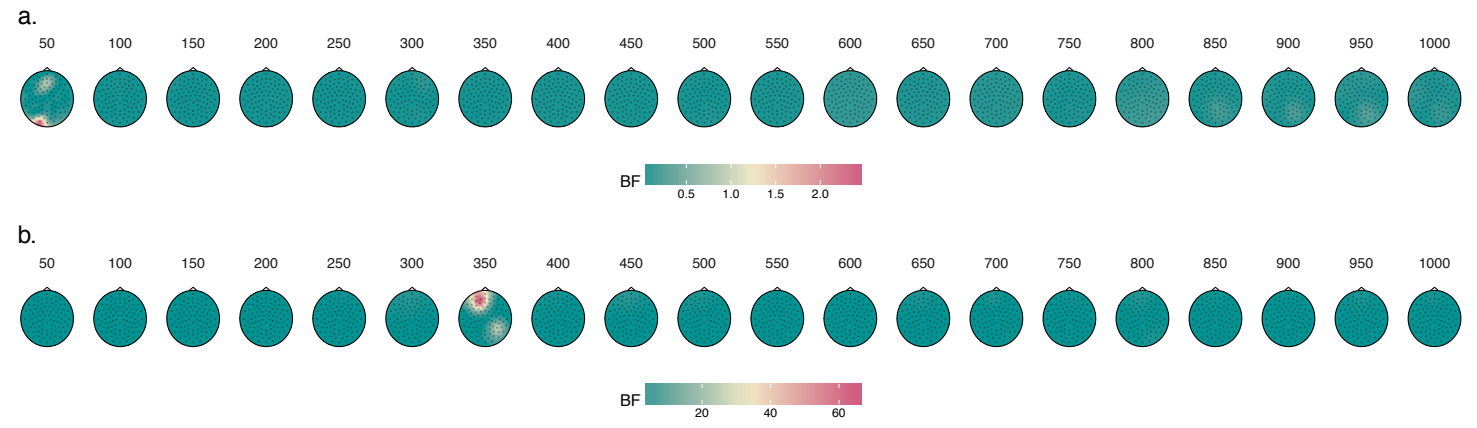

Figure 6. Effects of linguistic switch as a result of model comparisons in a. Step 2: models including linguistic switch vs Step 1: models excluding linguistic switch and b. Step 3: models including the interaction between linguistic switch and target modality vs Step 2: models excluding the interaction. BF $>3$ constitutes substantial evidence in favour of linguistic switching costs; $\mathrm{BF}>10$ constitutes strong evidence in favour or linguistic switching costs; BF > 100 constitutes decisive evidence in favour of linguistic switching costs.

of Step 3 models). We found that perceptual switch and linguistic switch performed equally well between $0 \mathrm{~ms}$ and $50 \mathrm{~ms}$ in the left occipital region (e.g., E70: $B F_{P L 2}=$ 2.91). The data supported neither model more. However, the data supported the interaction between linguistic switch and target modality more than the interaction between perceptual switch and target modality at the left frontal region (Figure 7b; e.g., E19: $\left.B F_{P L 3}=0.17\right)$ and the right parietal region $\left(\mathrm{E} 92: B F_{P L 3}=0.11\right)$ between $300 \mathrm{~ms}$ and $350 \mathrm{~ms}$. That is to say, by comparing the interaction models alone, linguistic switching costs were more than 5.88 times more likely than perceptual switching costs. It suggested that the "early N400 effect" found in previous studies was better explained by a switch between the linguistic dimensions than the perceptual modalities. Nevertheless, the fact that the linguistic switching costs were moderated by the target modalities suggested that modality-specific resources were also recruited to construct the conceptual representations. 


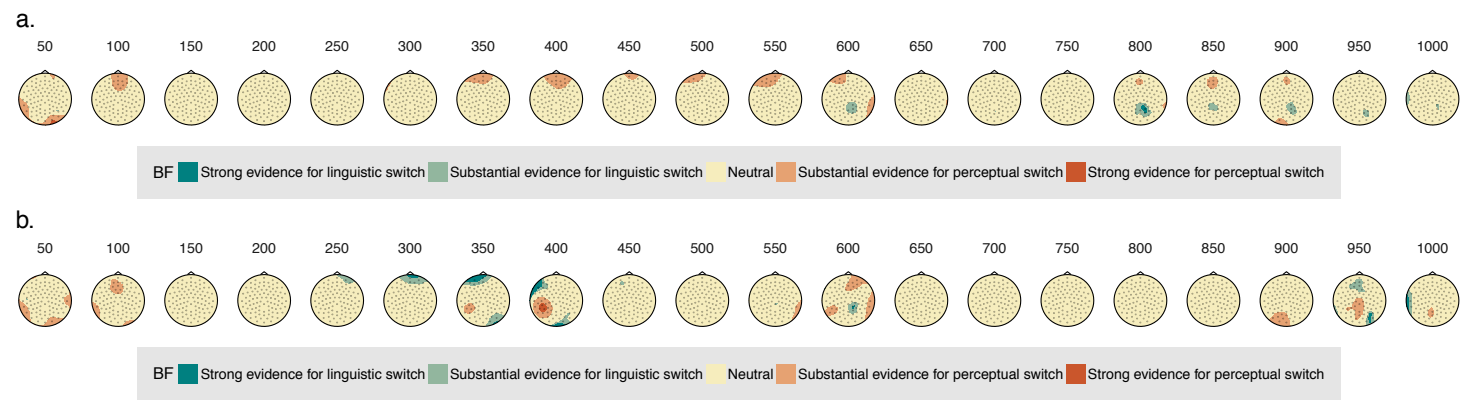

Figure 7. Evidence for perceptual switch in comparison with linguistic switch as a result of comparing models in a. Step 2 (excluding the interaction) and b. Step 3 (including the interaction). $B F_{P L}>3$ constitutes substantial evidence in favour of perceptual switching costs; $B F_{P L}>10$ constitutes strong evidence in favour or perceptual switching costs; $B F_{P L}<0.33$ constitutes substantial evidence in favour of linguistic switching costs; $B F_{P L}<0.1$ constitutes strong evidence in favour or linguistic switching costs.

Further exploration showed great distinction between the Step 2 models and Step 3 models. For example, the ERP in the frontal region around 350ms better supported the perceptual model in Step 2, but supported the interaction between linguistic switch and target modality in Step 3. This pattern suggested that it is the target modality that drove the difference between perceptual and linguistic switching costs. Therefore, we further analysed the data per target modality in the next section.

\section{Moderation of target modality.}

We performed hierarchical model comparisons again per target modality. That is,

1. For the perceptual switching costs, models were built in two steps.

Step $1_{\mathrm{P}}$ : Models with only random variables;

Step $2_{\mathrm{P}}$ : Models with random variables and perceptual switch. 
2. linguistic switch, models were built in two steps.

Step $1_{\mathrm{L}}$ : Models with only random variables;

Step $2_{\mathrm{L}}$ : Models with random variables and linguistic switch.

Since for auditory targets perceptual and linguistic switching conditions were exactly the same, two sets of hierarchical steps above produced the same results.

Auditory targets.

Evidence supported a switching effect at the onset of the epoch (within 50ms) and between $300 \mathrm{~ms}$ and $350 \mathrm{~ms}$ (Figure 8a), although the pattern of regression coefficients was consistent throughout the whole epoch (Figure 8b). Switch trials evoked larger positivity in the left frontal region and larger negativity in the right parietal and occipital regions. Since linguistic switch completely overlapped with perceptual switch, it could be argued that when the linguistic and perceptual switch happened simultaneously, it could be detected between 300ms and 350ms, coinciding with the early N400 effect from previous studies.

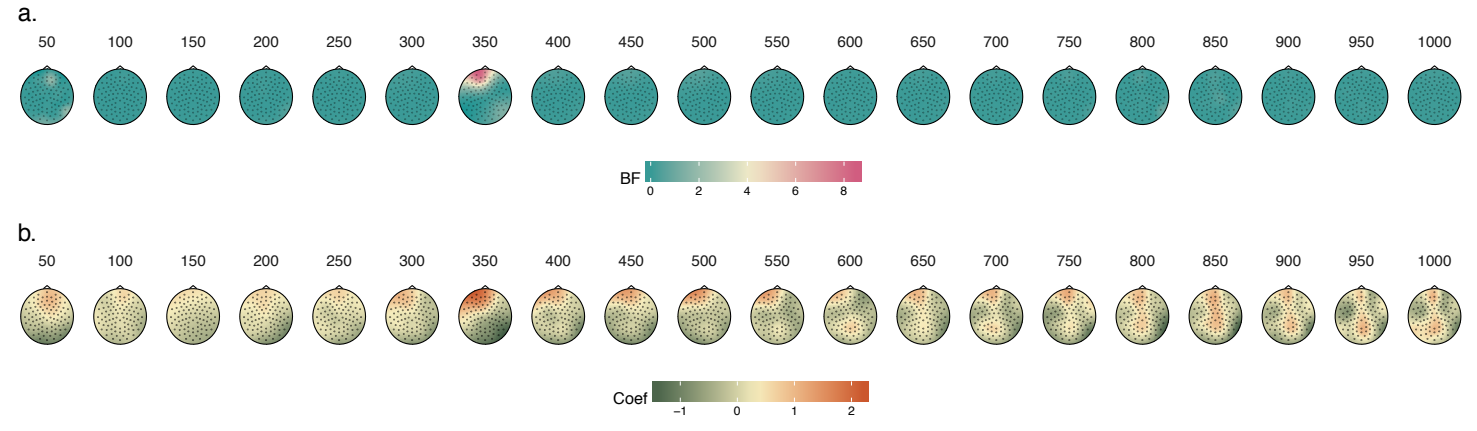

Figure 8. Switching costs for auditory targets illustrated by a. BF of models with perceptual-linguistic switch (Step 2) against models with only random variables (Step 1), and b. raw regression coefficients of perceptual-linguistic switch in Step 2 models.

Visual targets. 
For visual targets, it was possible to distinguish between perceptual switch and linguistic switch because half of the perceptual switch trials were linguistic non-switch, while the other half were linguistic switch. From Figure 9a, we found evidence against both linguistic and perceptual switching costs between 300ms-350ms, suggesting that the interaction effect in this time window was driven by the effect for auditory targets (and the lack thereof for visual targets). Furthermore, there was strong evidence against any perceptual switching costs for visual targets over the effect of random variables. Linguistic switch (Figure 9b) had an effect at the $800 \mathrm{~ms}$ slice in the right posterior regions (e.g., E84; $b=2.08, B F_{21 L v}$ $=4.32$ ), which meant that the linguistic switch evoked a greater positivity at this later stage of processing, corresponding to LPC which was often associated with decision making and conflict resolution.

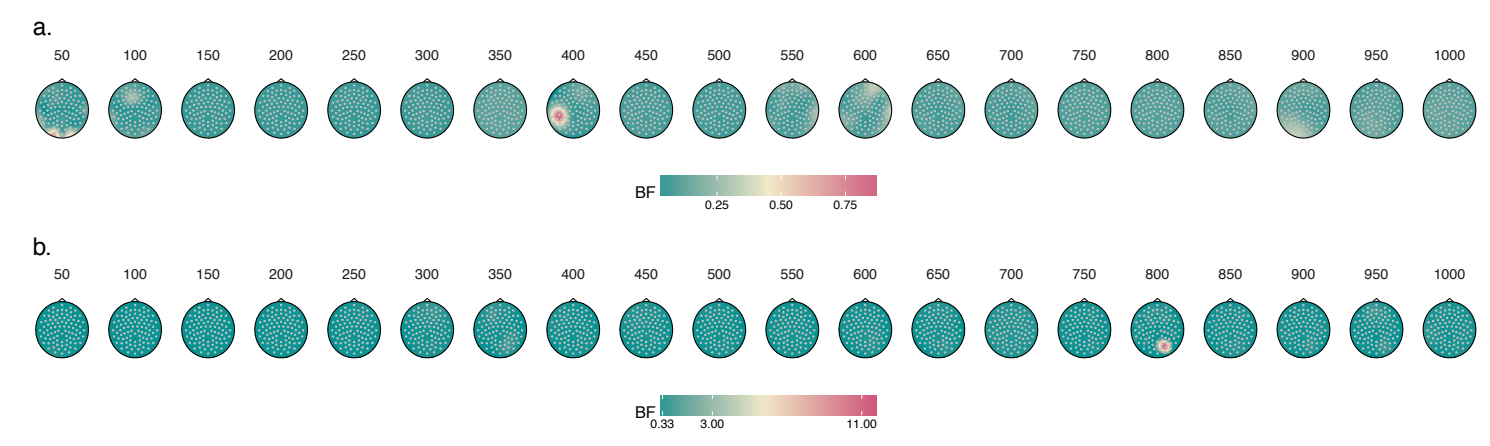

Figure 9. Effects of a. perceptual and b. linguistic switching costs for visual targets.

Again to show the relative power of perceptual and linguistic switch, Figure 10 is a topoplot of BFs by comparing the Step 2 models of visual targets, which was similar to Figure 7b. Strong evidence supported the linguistic switching costs in the frontal and occipital areas from $200 \mathrm{~ms}$ to $400 \mathrm{~ms}$. The linguistic switch evoked a stronger negativity in the frontal region (e.g., at 250ms-300ms, E 18: $b=-1.17$, $\left.B F_{P L v}=0.08\right)$, while it evoked a stronger positivity in the parietal region (e.g., at 300ms-350ms, E77: $\left.b=1.20, B F_{P L v}=0.10\right)$. This effect was consistent with the early N400 effect found for auditory targets, and the linguistic switch could be 
detected as early as 200ms. Again around 600ms, linguistic switch evoked a stronger positivity in the parietal region (e.g., at $600 \mathrm{~ms}-650 \mathrm{~ms}$, E77: $b=1.51, B F_{P L v}=$ 0.04), corresponding with P600 or LPC.

In contrast, perceptual switch started to have a stronger effect between 300ms and $400 \mathrm{~ms}$ in the left parietal region (e.g., E52: $b=-1.10, B F_{P L v}=14.77$ ). The results showed that perceptual switch evoked a stronger negativity than non-switch in this left-parietal region, corresponding with the classic N400 effect. Later around 600ms, perceptual switch had greater power than linguistic switch in the right temporal region (e.g., E108: $\left.b=-1.24, B F_{P L v}=10.95\right)$, meaning that perceptual switch elicited a stronger negativity than non-switch, which was the reverse of LPC.

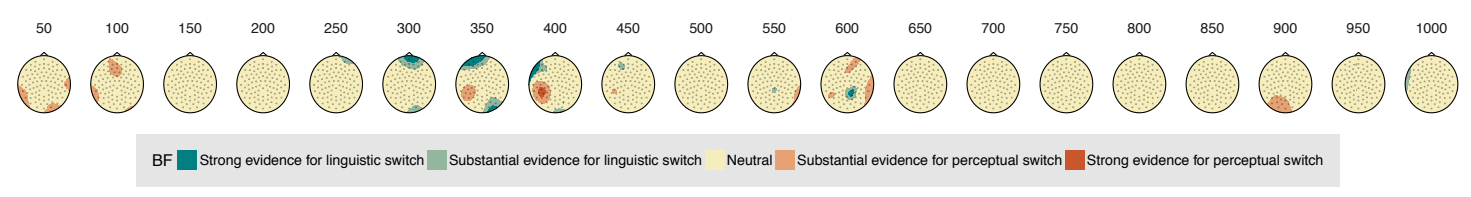

Figure 10. Evidence for perceptual switch in comparison with linguistic switch as a result of comparing models for visual targets in a. Step 2 (excluding the interaction) and b. Step 3 (including the interaction). $B F_{P L}>3$ constitutes substantial evidence in favour of perceptual switching costs; $B F_{P L}>10$ constitutes strong evidence in favour or perceptual switching costs; $B F_{P L}<0.33$ constitutes substantial evidence in favour of linguistic switching costs; $B F_{P L}<0.1$ constitutes strong evidence in favour or linguistic switching costs.

\section{Summary of ERP results.}

In summary, evidence supported both types of switch between $0 \mathrm{~ms}$ and $50 \mathrm{~ms}$ in the left occipital region, but the effects diverged later in the epoch. We found evidence for perceptual switching costs around 400ms in the left parietal region, and later around $600 \mathrm{~ms}$ in the right temporal region, albeit this effect only existed for visual targets and was relative to the power of linguistic switch. We also found 
evidence for linguistic switching costs around 300ms in the frontal region and parietal region, prior to the effect of the perceptual switch as predicted.

Furthermore, we found evidence for both linguistic and perceptual switch at the later stage of processing (after 600ms).

\section{General Discussion}

In this paper, our aim was to differentiate the contribution of linguistic dimension switch from perceptual modality switch in the modality switching costs, and thus to identify the neuroelectrical activations of simulation and linguistic information during conceptual representation. We found that what was considered to be the modality switching costs in ERP components, could be better explained by the switch between different linguistic distributional clusters, rather than the switch between perceptual modalities. Such a finding supported the idea that the linguistic component plays a crucial role, even bearing the burden of semantic representation (Connell, 2018). Because the activation of the linguistic component is more speedy than the embodied component as shown in the ERP, it was possible for the linguistic component to be a shortcut for the more computationally heavy and slower embodied simulation (Barsalou et al., 2008; Connell \& Lynott, 2014; Louwerse \& Jeuniaux, 2010)

An important finding in the present study was that words pertaining to different sensory modalities elicited different behavioural and neural responses. The behavioural study did not replicate previous findings, but showed that response time in the property verification task was not best explained by perceptual modality switching costs (i.e., loud $\rightarrow$ *bright* takes longer than red $\rightarrow$ *bright* because of switch from audition to vision); nor was it best explained by linguistic switch alone (i.e., switch from one linguistic dimension to another). Rather, it was best explained by the interaction between a switch between linguistic dimensions and the 
perceptual modality of the target word (which was slightly better than the model including the interaction between perceptual switch and the perceptual modality). In other words, the linguistic dimension switching costs occurred for words of some modalities (auditory and haptic words specifically) but not other words (visual words specifically).

The ERP results also showed the same pattern, that is: auditory words turned out to be the one category that elicited consistent modality switching costs, while visual and haptic words did not (see also Moscoso Del Prado Martín, Hauk, \& Pulvermüller, 2006; Schmidt-Snoek, Drew, Barile, \& Agauas, 2015). It could be best explained by the fact that visual and haptic words often belonged to the same linguistic dimension (Louwerse \& Connell, 2011), which is more coarse-grained than perceptual modalities. For auditory words, there was a complete overlap between the perceptual switch and the linguistic switch, whereas for haptic and visual words, there was a mismatch which caused different activations in the embodied versus the linguistic components. As a result, the difference between the two components needs to be reconciled, as shown by the effect of perceptual switch at $800 \mathrm{~ms}$ for the visual targets. These findings did not reject the idea of previous studies that processing sensory property words required modality-specific resources (Pecher et al., 2003). However, it supported the idea from a different angle. The linguistic symbols were activated first, triggering the simulation of sensory experiences of different modalities at various stage of processing. In this sense, the current finding improved on our previous understanding of embodied simulation, and showed the crucial function of linguistic distributional pattern as a guide for embodied simulation (Connell, 2018).

Our ERP results further supported the roles of the two components (linguistic component especially) we suggested. Confirming the theories such as LASS (Barsalou et al., 2008) and SIH (Louwerse, 2011), the linguistic component started to be activated before the embodied component from about $200 \mathrm{~ms}$, and reached the 
peak of its activation between $300 \mathrm{~ms}$ and $350 \mathrm{~ms}$. Such activation coincided with the early N400 effect found in previous studies on modality switching costs (Bernabeu et al., 2017; Hald et al., 2011). This effect of linguistic switch was the most prominent effect throughout the whole epoch, which supported our claim that what was considered perceptual modality switching costs was due to a switch between linguistic clusters. Starting from $300 \mathrm{~ms}$, the embodied component was activated, which peaked around 400ms, consistent with the classic N400 effect. Although this effect was relatively weaker than the effect of the linguistic component previously, it suggested that the embodied component was important to the construction of semantic representations (Kutas \& Federmeier, 2011). After 600ms, both embodied and linguistic components were active until the end of the epoch, suggesting that both components were involved in the decision making. This late effect (i.e., P600 or LPC) has been associated with conflict resolution for decision making (Polich, 2007) or integrating distinct conceptual representations (Davenport \& Coulson, 2011; Kuperberg, 2007; Paczynski \& Kuperberg, 2012). It could mean that even at the later stage of processing, the different information from embodied simulation and linguistic distributional patterns needs to be integrated and reconciled in order to construct a coherent representation of meaning.

Our study could not implement a full-fledged independent manipulation of the linguistic component because auditory words tend not to appear in the same linguistic cluster as other sensory properties (Louwerse \& Connell, 2011). If future studies could ensure independent factorial manipulation (i.e., both perceptual and linguistic switch, perceptual switch and linguistic non-switch, perceptual non-switch and linguistic switch, both perceptual and linguistic non-switch), we would predict that the early N400 effect would appear for the two conditions where there is a linguistic switch whereas the LPC to appear for the two conditions where there was a mismatch between perceptual and linguistic switch. 
Our study also found an immediate effect of both embodied and linguistic switch at the first 50ms of the epoch. Although embodied simulation and linguistic distributional patterns were found to have speedy activations, it was unlikely that they could affect semantic processing at the immediate property onset. Instead, this effect could be a result of spill-over from the concept word. Because a trial was presented with a concept (e.g., $S U N$ ) first for $500 \mathrm{~ms}$ followed by a property (e.g., bright), it was plausible that the conceptual representation of the concept word was still ongoing by the time the epoch started. Because the properties were usually salient for the concepts, the switching costs might have already been incurred when participants were processing the concept word. Bernabeu et al. (2017), in their recent replication of previous ERP studies, reversed the order of concept and property and did not find an immediate effect from the property onset. In future studies, it is recommended to adopt the reverse order, control the concept words so that they do not automatically activate the properties before the words are shown, or analyse epochs after both the concept and property words.

\section{Conclusion}

The present study provided support for the crucial role of the linguistic component in conceptual representations. Instead of finding it to perform a peripheral role, we found that it bore the burden of conceptual representations, preceding and even overpowering the effect of the embodied component. We suggest that representing the meaning of a word is indeed embodied, but the linguistic information support conceptual representation in an important way (Connell, 2018). The timecourse of the activations of the two components satisfies the requirement for the linguistic component to be a shortcut before the slower and more detailed simulation produces a detailed representation (Barsalou et al., 2008; Connell \& Lynott, 2014; Louwerse \& Jeuniaux, 2010). 


\section{References}

Amsel, B. D. (2011). Tracking real-time neural activation of conceptual knowledge using single-trial event-related potentials. Neuropsychologia, 49(5), 970-983. doi:10.1016/j.neuropsychologia.2011.01.003

Andrews, M., Vigliocco, G., \& Vinson, D. (2009). Integrating experiential and distributional data to learn semantic representations. Psychological Review, 116(3), 463-498. doi:10.1037/a0016261

Auguie, B. (2017). GridExtra: Miscellaneous functions for "grid" graphics. Retrieved from https://CRAN.R-project.org/package=gridExtra

Aust, F., \& Barth, M. (2017). papaja: Create APA manuscripts with R Markdown. Retrieved from https://github.com/crsh/papaja

Bache, S. M., \& Wickham, H. (2014). Magrittr: A forward-pipe operator for $r$. Retrieved from https://CRAN.R-project.org/package=magrittr

Barsalou, L. W. (1999). Perceptual symbol systems. The Behavioral and Brain Sciences, 22(4), 577-609; discussion 610-60.

Barsalou, L. W., Santos, A., Simmons, W. K., \& Wilson, C. (2008). Language and simulation in conceptual processing. In A. M. Glenberg \& A. C. Graesser (Eds.), Symbols, embodiment, and meaning (pp. 245-283). Oxford, England: Oxford University Press.

Bates, D., \& Maechler, M. (2017). Matrix: Sparse and dense matrix classes and methods. Retrieved from https://CRAN.R-project.org/package=Matrix

Bates, D., Mächler, M., Bolker, B., \& Walker, S. (2015). Fitting linear mixed-effects models using lme4. Journal of Statistical Software, 67(1), 1-48. doi:10.18637/jss.v067.i01

Bernabeu, P., Willems, R. M., \& Louwerse, M. M. (2017). Modality switch effects 
emerge early and increase throughout conceptual processing: Evidence from ERPs. In 39th annual conference of cognitive science society. PsyArXiv.

Brants, T., \& Franz, A. (2006). Web 1T 5-gram Version 1 LDC2006T13. DVD. Philadelphia: Linguistic Data Consortium.

Chemero, A. (2009). Radical embodied cognitive science (p. 252). MIT Press.

Clark, H. H. (1973). The language-as-fixed-effect fallacy: A critique of language statistics in psychological research. Journal of Verbal Learning and Verbal Behavior, 12(4), 335-359. doi:10.1016/S0022-5371(73)80014-3

Collins, J., Pecher, D., Zeelenberg, R., \& Coulson, S. (2011). Modality Switching in a Property Verification Task: An ERP Study of What Happens When Candles Flicker after High Heels Click. Frontiers in Psychology, 2(February), 10. doi:10.3389/fpsyg. 2011.00010

Connell, L. (2018). What have labels ever done for us? The lingusitic shortcut in conceptual processing. Language, Cognition and Neuroscience. doi:10.1080/23273798.2018.1471512

Connell, L., \& Lynott, D. (2011). Modality Switching Costs Emerge in Concept Creation as Well as Retrieval. Cognitive Science, 35(4), 763-778. doi:10.1111/j.1551-6709.2010.01168.x

Connell, L., \& Lynott, D. (2013). Flexible and fast: linguistic shortcut affects both shallow and deep conceptual processing. Psychonomic Bulletin \& Review, 20(3), 542-50. doi:10.3758/s13423-012-0368-x

Connell, L., \& Lynott, D. (2014). Principles of representation: Why you can't represent the same concept twice. Topics in Cognitive Science, 6(3), 390-406. doi:10.1111/tops.12097

Dantzig, S. van, Cowell, R. A., Zeelenberg, R., \& Pecher, D. (2011). A sharp image 
or a sharp knife: norms for the modality-exclusivity of 774 concept-property items. Behavior Research Methods, 43(1), 145-54.

doi:10.3758/s13428-010-0038-8

Davenport, T., \& Coulson, S. (2011). Predictability and novelty in literal language comprehension: an ERP study. Brain Research, 1418, 70-82. doi:10.1016/j.brainres.2011.07.039

Dehaene, S., Naccache, L., Le Clec'H, G., Koechlin, E., Mueller, M., Dehaene-Lambertz, G., ... Le Bihan, D. (1998). Imaging unconscious semantic priming. Nature, 395(6702), 597-600. doi:10.1038/26967

Dowle, M., \& Srinivasan, A. (2017). Data.table: Extension of 'data.frame'. Retrieved from https://CRAN.R-project.org/package=data.table

Faul, F., Erdfelder, E., Buchner, A., \& Lang, A. G. (2009). Statistical power analyses using $\mathrm{G}^{*}$ Power 3.1: Tests for correlation and regression analyses. Behavior Research Methods, 41(4), 1149-1160. doi:10.3758/BRM.41.4.1149

Faul, F., Erdfelder, E., Lang, A. G., \& Buchner, A. (2007). G*Power: A flexible statistical power analysis program for the social, behavioral, and biomedical sciences. Behavior Research Methods, 39(2), 175-191. doi:10.3758/BF03193146

Firth, J. R. (1957). A synopsis of linguistic theory 1930-55. In Studies in linguistic analysis (special volume of the philological society) (Vols. 1952-59, pp. 1-32). Oxford, England: Blackwell Publishers.

Gelman, A., \& Su, Y.-S. (2018). Arm: Data analysis using regression and multilevel/hierarchical models. Retrieved from https://CRAN.R-project.org/package=arm

Genz, A., \& Bretz, F. (2009). Computation of multivariate normal and $t$ 
probabilities. Heidelberg: Springer-Verlag.

Hald, L. A., Hocking, I., Vernon, D., Marshall, J.-A., \& Garnham, A. (2013). Exploring modality switching effects in negated sentences: further evidence for grounded representations. Frontiers in Psychology, 4(February), 93. doi:10.3389/fpsyg.2013.00093

Hald, L. A., Marshall, J.-A., Janssen, D. P., \& Garnham, A. (2011). Switching Modalities in A Sentence Verification Task: ERP Evidence for Embodied Language Processing. Frontiers in Psychology, 2(March), 45. doi:10.3389/fpsyg.2011.00045

Harnad, S. (1990). The symbol grounding problem. Physica D: Nonlinear Phenomena, 42(1-3), 335-346. doi:10.1016/0167-2789(90)90087-6

Harrell Jr, F. E. (2018). Rms: Regression modeling strategies. Retrieved from https://CRAN.R-project.org/package $=$ rms

Harrell Jr, F. E., Charles Dupont, \& others. (2018). Hmisc: Harrell miscellaneous. Retrieved from https://CRAN.R-project.org/package=Hmisc

Hauk, O. (2016). Only time will tell - why temporal information is essential for our neuroscientific understanding of semantics. Psychonomic Bulletin $\&$ Review, 23(4), 1072-1079. doi:10.3758/s13423-015-0873-9

Hauk, O., Johnsrude, I., \& Pulvermüller, F. (2004). Somatotopic Representation of Action Words in Human Motor and Premotor Cortex. Neuron, 41(2), 301-307. doi:10.1016/S0896-6273(03)00838-9

Hauk, O., Pulvermüller, F., Ford, M., Marslen-Wilson, W. D., \& Davis, M. H. (2009). Can I have a quick word? Early electrophysiological manifestations of psycholinguistic processes revealed by event-related regression analysis of the EEG. Biological Psychology, 80(1), 64-74. 
doi:10.1016/j.biopsycho.2008.04.015

Hendrix, P., Bolger, P., \& Baayen, R. H. (2017). Distinct ERP signatures of word frequency, phrase frequency, and prototypicality in speech production. Journal of Experimental Psychology: Learning, Memory, and Cognition, 43(1), 128-149. doi:10.1037/a0040332

Henry, L., \& Wickham, H. (2018). Purrr: Functional programming tools. Retrieved from https://CRAN.R-project.org/package=purrr

Hothorn, T. (2017). TH.data: TH's data archive. Retrieved from https://CRAN.R-project.org/package=TH.data

Hothorn, T., Bretz, F., \& Westfall, P. (2008). Simultaneous inference in general parametric models. Biometrical Journal, 50(3), 346-363.

Højsgaard, S., \& Halekoh, U. (2016). DoBy: Groupwise statistics, lsmeans, linear contrasts, utilities. Retrieved from https://CRAN.R-project.org/package=doBy

Jarosz, A. F., \& Wiley, J. (2014). What are the odds? A practical guide to computing and reporting Bayes Factors. The Journal of Problem Solving, 7 , 2-9. doi:10.7771/1932-6246.1167

Jeffreys, H. (1998). Theory of probability (3rd ed.). Oxford, UK: Oxford University Press.

Kintsch, W., \& Dijk, T. V. (1978). Toward a model of text comprehension and production. Psychological Review, 85(5), 363-394.

Koenker, R., \& Ng, P. (2017). SparseM: Sparse linear algebra. Retrieved from https://CRAN.R-project.org/package=SparseM

Kuhn, M., Steve Weston, Wing, J., Forester, J., \& Thaler, T. (2016). Contrast: A collection of contrast methods. Retrieved from 
https://CRAN.R-project.org/package=contrast

Kuperberg, G. R. (2007). Neural mechanisms of language comprehension:

Challenges to syntax. Brain Research, 1146(1), 23-49.

doi:10.1016/j.brainres.2006.12.063

Kutas, M., \& Federmeier, K. D. (2011). Thirty years and counting: finding meaning in the N400 component of the event-related brain potential (ERP). Annual Review of Psychology, 62, 621-47. doi:10.1146/annurev.psych.093008.131123

Kuznetsova, A., Brockhoff, P. B., \& Christensen, R. H. B. (2017). lmerTest package: Tests in linear mixed effects models. Journal of Statistical Software, 82(13), 1-26. doi:10.18637/jss.v082.i13

Landauer, T. K., \& Dumais, S. T. (1997). A solution to Plato's problem: The latent semantic analysis theory of acquisition, induction, and representation of knowledge. Psychological Review, 104(2), 211-40.

doi:10.1037/0033-295X.104.2.211

Lebois, L. A. M., Wilson-Mendenhall, C. D., \& Barsalou, L. W. (2015). Are Automatic Conceptual Cores the Gold Standard of Semantic Processing? The Context-Dependence of Spatial Meaning in Grounded Congruency Effects. Cognitive Science, 39(8), 1764-1801. doi:10.1111/cogs.12174

Louwerse, M. M. (2011). Symbol interdependency in symbolic and embodied cognition. Topics in Cognitive Science, 3(2), 273-302. doi:10.1111/j.1756-8765.2010.01106.x

Louwerse, M. M., \& Connell, L. (2011). A taste of words: linguistic context and perceptual simulation predict the modality of words. Cognitive Science, 35(2), 381-98. doi:10.1111/j.1551-6709.2010.01157.x

Louwerse, M. M., \& Jeuniaux, P. (2008). Language comprehension is both embodied and symbolic. In A. Glenberg \& A. C. Graesser (Eds.), Embodiment and 
meaning: A debate (pp. 309-326). Oxford, England: Oxford University Press.

Louwerse, M. M., \& Jeuniaux, P. (2010). The linguistic and embodied nature of conceptual processing. Cognition, 114(1), 96-104.

doi:10.1016/j.cognition.2009.09.002

Luck, S. J. (2005). Ten Simple Rules for Deisgning ERP Experiments. In Event-related potentials: A methods handbook (pp. 17-32). doi:10.1371/journal.pcbi.0020012

Lynott, D., \& Connell, L. (2009). Modality exclusivity norms for 423 object properties. Behavior Research Methods, 41(2), 558-64. doi:10.3758/BRM.41.2.558

Morey, R. D., \& Rouder, J. N. (2015). BayesFactor: Computation of bayes factors for common designs. Retrieved from https://CRAN.R-project.org/package=BayesFactor

Moscoso Del Prado Martín, F., Hauk, O., \& Pulvermüller, F. (2006). Category specificity in the processing of color-related and form-related words: An ERP study. NeuroImage, 29(1), 29-37. doi:10.1016/j.neuroimage.2005.07.055

Nowosad, J. (2017). Rcartocolor: 'CARTOColors' palettes. Retrieved from https://CRAN.R-project.org/package=rcartocolor

Otten, L. J., \& Rugg, M. D. (2005). Interpreting event-related brain potentials. In T. C. Handy (Ed.), Event-related potentials: A methods handbook (pp. 3-16). Cambridge: MIT Press.

Öttl, B., Dudschig, C., \& Kaup, B. (2017). Forming associations between language and sensorimotor traces during novel word learning. Language and Cognition, 9(01), 156-171. doi:10.1017/langcog.2016.5

Paczynski, M., \& Kuperberg, G. R. (2012). Multiple influences of semantic memory 
on sentence processing: Distinct effects of semantic relatedness on violations of real-world event/state knowledge and animacy selection restrictions. Journal of Memory and Language, 67(4), 426-448. doi:10.1016/j.jml.2012.07.003

Pecher, D., Zeelenberg, R., \& Barsalou, L. W. (2003). Verifying different-modality properties for concepts produces switching costs. Psychological Science, $14(2), 119-24$.

Pinheiro, J., Bates, D., DebRoy, S., Sarkar, D., \& R Core Team. (2017). nlme: Linear and nonlinear mixed effects models. Retrieved from https://CRAN.R-project.org/package=nlme

Plate, T., \& Heiberger, R. (2016). Abind: Combine multidimensional arrays. Retrieved from https://CRAN.R-project.org/package=abind

Plummer, M., Best, N., Cowles, K., \& Vines, K. (2006). CODA: Convergence diagnosis and output analysis for mcmc. R News, 6(1), 7-11. Retrieved from https://journal.r-project.org/archive/

Polich, J. (2007). Updating P300: an integrative theory of P3a and P3b. Clinical Neurophysiology : Official Journal of the International Federation of Clinical Neurophysiology, 118(10), 2128-48. doi:10.1016/j.clinph.2007.04.019

R Core Team. (2017). R: A language and environment for statistical computing. Vienna, Austria: R Foundation for Statistical Computing. Retrieved from https://www.R-project.org/

Revelle, W. (2018). Psych: Procedures for psychological, psychometric, and personality research. Evanston, Illinois: Northwestern University. Retrieved from https://CRAN.R-project.org/package=psych

Robinson, D. (2017). Broom: Convert statistical analysis objects into tidy data 
frames. Retrieved from https://CRAN.R-project.org/package=broom

Sarkar, D. (2008). Lattice: Multivariate data visualization with $r$. New York: Springer. Retrieved from http://lmdvr.r-forge.r-project.org

Schmidt-Snoek, G. L., Drew, A. R., Barile, E. C., \& Agauas, S. J. (2015). Auditory and motion metaphors have different scalp distributions: an ERP study. Frontiers in Human Neuroscience, 9(March), 1-9. doi:10.3389/fnhum.2015.00126

Searle, J. R. (1980). Minds, brains, and programs. The Behavioral and Brain Sciences, 3(3), 417-457. doi:10.1017/S0140525X00005756

Simmons, J. P., Nelson, L. D., \& Simonsohn, U. (2011). False-positive psychology: Undisclosed flexibility in data collection and analysis allows presenting anything as significant. Psychological Science, 22(11), 1359-1366. doi:10.1177/0956797611417632

Solomon, K. O., \& Barsalou, L. W. (2004). Perceptual simulation in property verification. Memory \& Cognition, 32(2), 244-259. doi:10.3758/BF03196856

Solt, F., \& Hu, Y. (2015). interplot: Plot the effects of variables in interaction terms. Available at The Comprehensive R Archive Network (CRAN). Retrieved from https://CRAN.R-project.org/package=interplot

Terry M. Therneau, \& Patricia M. Grambsch. (2000). Modeling survival data: Extending the Cox model. New York: Springer.

Van Dantzig, S., Pecher, D., Zeelenberg, R., \& Barsalou, L. W. (2008). Perceptual processing affects conceptual processing. Cognitive Science, 32(3), 579-90. doi:10.1080/03640210802035365

Venables, W. N., \& Ripley, B. D. (2002). Modern applied statistics with s (Fourth.). 
New York: Springer. Retrieved from http://www.stats.ox.ac.uk/pub/MASS4

Wagenmakers, E.-J. (2007). A practical solution to the pervasive problems of $\mathrm{p}$ values. Psychonomic Bulletin \& Review, 14(5), 779-804. doi:10.3758/BF03194105

Wickham, H. (2009). Ggplot2: Elegant graphics for data analysis. Springer-Verlag New York. Retrieved from http://ggplot2.org

Wickham, H. (2011). The split-apply-combine strategy for data analysis. Journal of Statistical Software, 40(1), 1-29. Retrieved from http://www.jstatsoft.org/v40/i01/

Wickham, H., \& Henry, L. (2017). Tidyr: Easily tidy data with 'spread()' and 'gather()' functions. Retrieved from https://CRAN.R-project.org/package=tidyr

Wickham, H., Francois, R., Henry, L., \& Müller, K. (2017). Dplyr: A grammar of data manipulation. Retrieved from https://CRAN.R-project.org/package=dplyr

Wood, S. N. (2003). Thin-plate regression splines. Journal of the Royal Statistical Society (B), 65(1), 95-114.

Wood, S. N. (2004). Stable and efficient multiple smoothing parameter estimation for generalized additive models. Journal of the American Statistical Association, 99(467), 673-686.

Wood, S. N. (2011). Fast stable restricted maximum likelihood and marginal likelihood estimation of semiparametric generalized linear models. Journal of the Royal Statistical Society (B), 73(1), 3-36.

Wood, S., N., Pya, \& S"afken, B. (2016). Smoothing parameter and model selection for general smooth models (with discussion). Journal of the American 
Statistical Association, 111, 1548-1575.

Xie, Y. (2015). Dynamic documents with $R$ and knitr (2nd ed.). Boca Raton,

Florida: Chapman; Hall/CRC. Retrieved from https://yihui.name/knitr/

Xie, Y. (2016). Bookdown: Authoring books and technical documents with $R$ markdown. Boca Raton, Florida: Chapman; Hall/CRC. Retrieved from https://github.com/rstudio/bookdown

Zeileis, A., \& Croissant, Y. (2010). Extended model formulas in R: Multiple parts and multiple responses. Journal of Statistical Software, 34(1), 1-13. doi:10.18637/jss.v034.i01

Zwaan, R. A. (2014). Embodiment and language comprehension: Reframing the discussion. Trends in Cognitive Sciences, 18(5), 229-234. doi:10.1016/j.tics.2014.02.008 
Appendix

128-channel EEG net layout

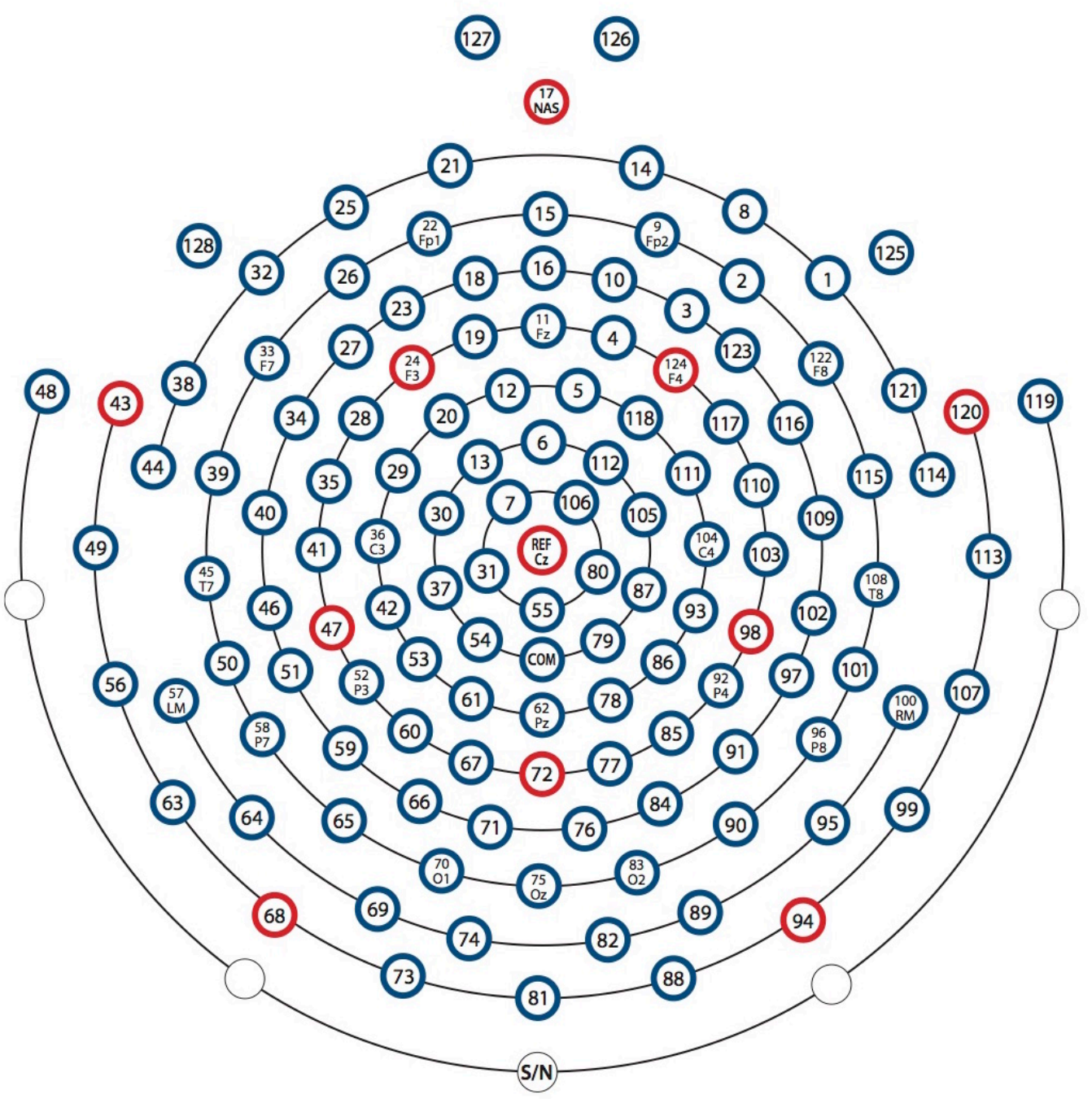

Figure A1. 128-channel EEG net layout 